\title{
Spectral Geometry in the Presence of Symmetry
}

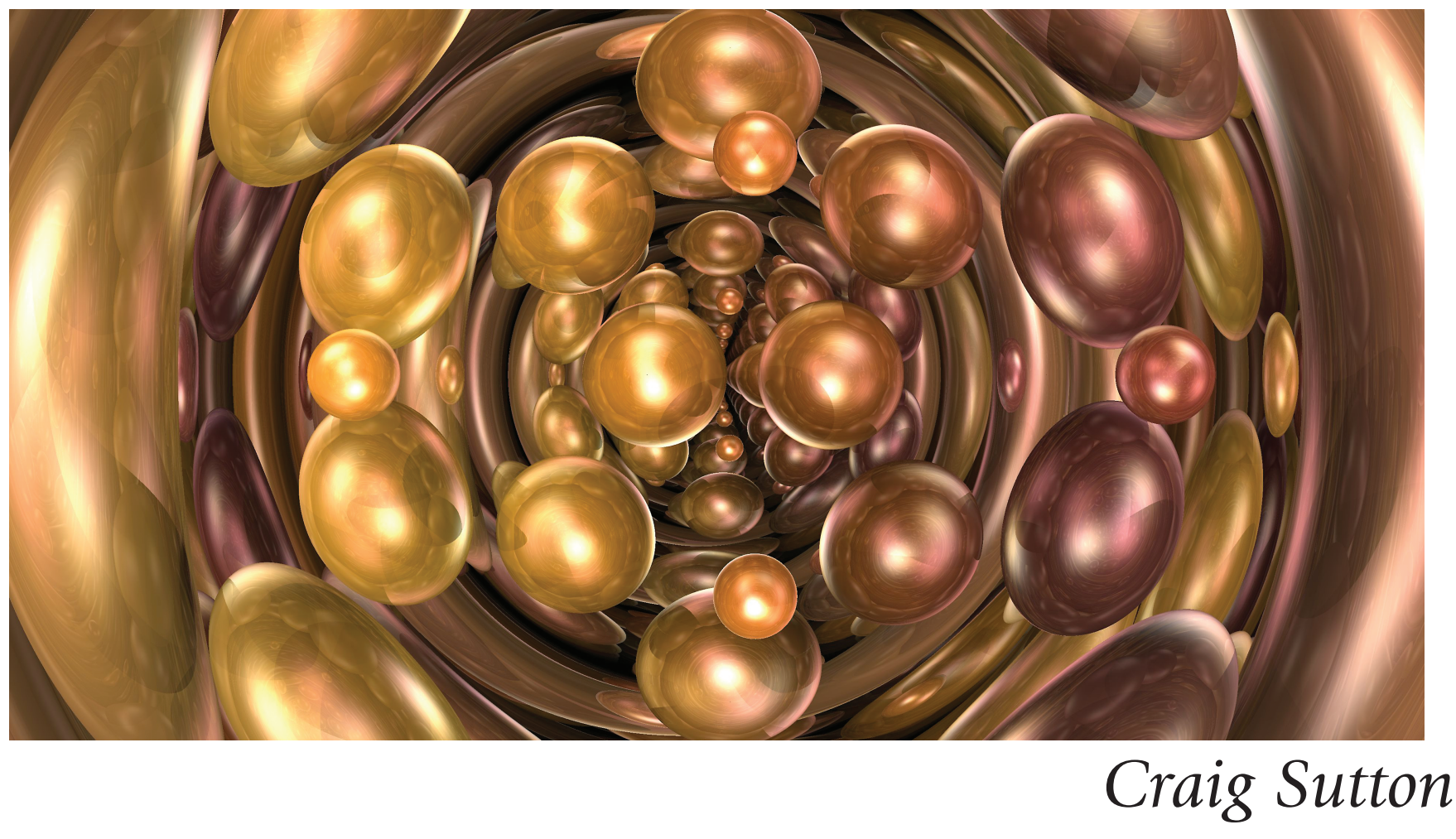

Let's play a game! Suppose there is a screen separating us. On my side of the screen there is a drum and on your side of the screen there are materials from which you can build a drum. After listening to me play my drum, your task is to build one that "sounds" identical to mine. (For the purposes of this thought experiment we'll assume you have perfect pitch and are an expert drum maker!) When your drum is complete, the screen is lifted and we consider the degree to which the geometry of our drums are necessarily identical. For instance, in order for your drum to sound like mine, does it necessarily have the same surface area as my drum? Or, in order for our drums to sound alike, are

Craig Sutton is an associate professor of mathematics, Director of the E.E. Just Program, and House Professor of School House at Dartmouth College. His email address is craig.j.sutton@dartmouth.edu.

This article is dedicated to my parents and written in solidarity with the Black Lives Matter movement.

Communicated by Notices Associate Editor Chikako Mese.

For permission to reprint this article, please contact:

reprint-permission@ams.org.

DOI: https://doi.org/10.1090/noti2294 they required to have the same "curviness"? In brief, we wonder about the extent to which it is possible to hear the shape of a drum.

This whimsical question was the basis for Mark Kac's highly influential article "Can one hear the shape of a drum?" [Ka]. Written a little over half a century ago, Kac's article helped spark renewed interest in the inverse spectral problem. To formulate Kac's question in precise mathematical terms, let $\Omega \subset \mathbb{R}^{2}$ be a connected bounded domain, which we can think of as a drumhead that vibrates in the direction orthogonal to the plane. If we let $u(x, y, t)$ denote the (orthogonal) displacement of the point $(x, y) \in$ $\Omega$ at time $t$ after the drumhead has been struck, then $u$ satisfies the wave equation:

$$
\frac{\partial^{2} u}{\partial t^{2}}+\Delta u=0 \text { with } u \uparrow \partial \Omega \equiv 0,
$$

where $\Delta=-\left(\frac{\partial^{2}}{\partial x^{2}}+\frac{\partial^{2}}{\partial y^{2}}\right)$ is the Laplace operator (with the given boundary conditions), a self-adjoint operator on $L^{2}(\Omega)$. The spectrum of $\Omega$ is defined to be the eigenvalues $\lambda_{0}=0<\lambda_{1} \leq \lambda_{2} \leq \cdots \leq \lambda_{k} \nearrow+\infty$ of the associated Laplace operator on $L^{2}(\Omega)$, and two domains are said to 

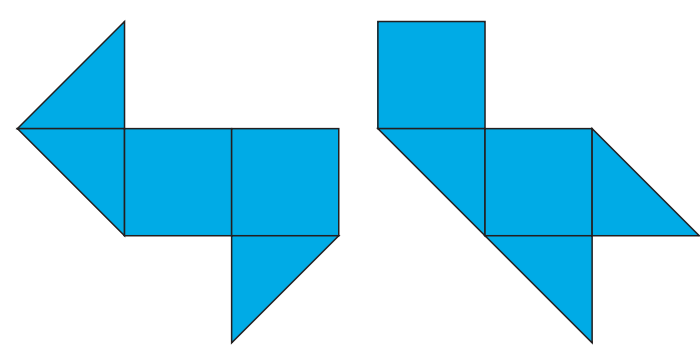

Figure 1. Isospectral planar domains of Gordon, Webb, and Wolpert [GWW].

be isospectral if their spectra agree. Finally, a geometric property that must be shared by two isospectral domains is a spectral invariant or, in the spirit of the metaphor, is said to be audible.

Using separation of variables, the general solution to equation (1) is given by

$$
u(x, y, t)=\sum_{k=0}^{\infty}\left(a_{k} \cos \left(\sqrt{\lambda_{k}} t\right)+b_{k} \sin \left(\sqrt{\lambda_{k}} t\right)\right) \varphi_{k}(x, y),
$$

where $a_{k}, b_{k} \in \mathbb{R}$ are constants and $\left\langle\varphi_{k}\right\rangle_{k=0}^{\infty}$ is an orthonormal basis of $L^{2}(\Omega)$ consisting of $\Delta$-eigenfunctions: $\Delta \varphi_{k}=\lambda_{k} \varphi_{k}$. Therefore, the spectrum of $\Omega$ is equivalent to knowing the natural frequencies $\left\langle\frac{\sqrt{\lambda_{k}}}{2 \pi}\right\rangle_{k=0}^{\infty}$ at which the drumhead will freely vibrate: these frequencies are the overtones of the drumhead. Now, the question posed in the title of Kac's article can be phrased as follows.

Problem 1. Are two isospectral bounded domains in $\mathbb{R}^{2}$ necessarily isometric?

Kac has shown that the spectrally determined analytic function $\sum_{k=0}^{\infty} e^{-\lambda_{k} t}$ on $\mathbb{R}_{+} \equiv(0,+\infty)$ admits the following asymptotic expansion:

$$
\sum_{k=0}^{\infty} e^{-\lambda_{k} t} \stackrel{t \precsim 0}{\sim} \frac{\operatorname{vol}(\Omega)}{2 \pi t}-\frac{L(\partial \Omega)}{4 \sqrt{2 \pi t}},
$$

where $\operatorname{vol}(\Omega)$ and $L(\partial \Omega)$ represent the volume and boundary length of $\Omega$, respectively [Ka]. Consequently, volume and boundary length are audible properties of planar domains, and, recalling that $L(\partial \Omega) \geq 4 \pi \operatorname{vol}(\Omega)$ with equality if and only if $\Omega$ is a disk, it follows that the disk of radius $R$-the planar domain with the "largest" symmetry group-is determined up to isometry by its spectrum (cf. Problem 2). In spite of this evidence in support of an affirmative answer to Problem 1, Gordon, Webb, and Wolpert were able to construct examples of isospectral yet non-isometric bounded planar domains having polygonal boundaries [GWW]. ${ }^{1}$

\footnotetext{
${ }^{1}$ It is still an open question whether there are examples of non-isometric isospectral planar domains with smooth boundaries. See [Ze] for a discussion of recent results on this question.
}

While there is more that can be said about the spectral geometry of planar domains (see [Ze], for example), the present survey will largely concern itself with the generalization of Problem 1 to $n$-dimensional closed manifolds with an emphasis on the role symmetry has played in our understanding of inverse spectral geometry. In particular, in section 2, we will discuss how symmetry can be harnessed to construct isospectral manifolds, emphasizing construction techniques that yield isospectral simply-connected Riemannian manifolds as found in [Sc, Go2, Sut, AYY]. And, in section 3, we will explore our ability to "hear" the geometry of spaces with "large" symmetry groups, focusing on the author's recent work with Samuel Lin and Ben Schmidt regarding the audibility of the Thurston geometries and, more generally, threedimensional geometric structures [LSS1, LSS2].

\section{The General Setup}

The Laplace operator on a Riemannian manifold $(M, g)$ is defined to be

$$
\Delta_{g}=-\operatorname{div}_{g} \circ \operatorname{grad}_{g}: C^{\infty}(M) \rightarrow C^{\infty}(M),
$$

where $\operatorname{grad}_{\mathrm{g}}$ assigns to any smooth function $f$ its gradient vector field $\operatorname{grad} f$ :

$$
d f_{p}(v)=g_{p}(v, \operatorname{grad} f(p))
$$

for any $p \in M$ and $v \in T_{p} M$, and $\operatorname{div}_{g}$ assigns to each smooth vector field $X$ its divergence $\operatorname{div}_{g} X$, a smooth function that measures the infinitesimal distortion of volume under $\Phi_{t}$, the flow of $X$ :

$$
v^{\prime}(0)=\int_{K} \operatorname{div}_{g} X d \operatorname{vol}_{g},
$$

where $v(t)=\int_{\Phi_{t}(K)} d \operatorname{vol}_{g}$ and $K$ is a compact set. As before, $\Delta_{g}$ is extended to an (essentially) self-adjoint operator on $L^{2}(M)$ with a discrete spectrum $\lambda_{0}=0<\lambda_{1} \leq$ $\lambda_{2} \leq \cdots \leq \lambda_{k} \nearrow+\infty$ accumulating only at infinity. This sequnce of eigenvalues is known as the spectrum of the manifold $(M, g)$ and will be denoted $\operatorname{Spec}_{\Delta}(M, g)$. On occasion it will be useful to consider the eigenvalue set of the Laplace operator $\mathscr{E}_{\Delta}(M, g)$, which is the sequence $\mu_{0}=0<\mu_{1}<\mu_{2}<\cdots<\mu_{k} \nearrow+\infty$ consisting of the eigenvalues of the Laplace operator ignoring their multiplicities. And, for any $\lambda \in \mathscr{E}(M, g)$ we let $L^{2}\left(M, v_{g}\right)_{\lambda}$ denote the corresponding eigenspace. Two manifolds are said to be isospectral if their spectra agree.

The most fundamental geometric invariant of a Riemannian manifold $(M, g)$ is sectional curvature, which measures the rate at which the geodesics at a point spread relative to geodesics in Euclidean space. The geodesics in the Euclidean plane $\mathbb{E}^{2}$ are straight lines and the straight lines emanating from a fixed point spread linearly, as is reflected by the fact that any circle of radius $r>0$ in $\mathbb{E}^{2}$ has 
length $2 \pi r$. Now, given a point $p$ in a Riemannian manifold $(M, g)$ and a two-dimensional subspace $\sigma \leq T_{p} M$, the sectional curvature of $\sigma$, denoted $\operatorname{Sec}(\sigma)$, is the scalar defined by the Taylor expansion

$$
L\left(C_{r}\right)=2 \pi r-\frac{\operatorname{Sec}(\sigma)}{6} r^{3}+o\left(r^{5}\right),
$$

where $L\left(C_{r}\right)$ is the length of $C_{r}$, the circle of radius $r$ centered at $p$ and determined by the geodesics $\gamma$ with initial condition $\gamma^{\prime}(0)$ in $\sigma$. Therefore, if $\operatorname{Sec}(\sigma)$ is positive (respectively, zero or negative), then the geodesics determined by $\sigma$ initially spread sublinearly (respectively, linearly or superlinearly). The sectional curvature of a Riemannian manifold $(M, g)$ is conveniently encoded in its Riemannian curvature tensor $R$, a $(4,0)$-tensor associated to $(M, g)$. Other notions of curvature include the Ricci curvature tensor, denoted by Ric, and the scalar curvature function, denoted by Scal, which measure the degree to which the growth of the volume of small cones and balls in $(M, g)$ differ from their Euclidean counterparts, respectively.

To find relationships between the geometry of $(M, g)$ and its spectrum, it has proven fruitful to examine the singularities of various spectrally determined distributions by probing their attendant asymptotic expansions for geometric data. In particular, it is useful to analyze the singularities of

1. (Heat Trace) $\operatorname{Tr}\left(e^{-t \Delta_{g}}\right) \equiv \sum_{k=0}^{\infty} e^{-t \lambda_{k}}$,

2. (Wave Trace) $\operatorname{Tr}\left(e^{-i t \sqrt{\Delta_{g}}}\right) \equiv \sum_{k=0}^{\infty} e^{-i t \sqrt{\lambda_{k}}}$.

The heat trace is an honest analytic function on the positive real line that approaches infinity as $t$ nears zero, while the wave trace is a distribution on $\mathbb{R}$ whose singularities (up to sign) can only occur at lengths of smoothly closed geodesics. This article will only make use of the heat trace. For a survey that includes applications of the wave trace, we recommend $[\mathrm{Ze}]$.

It is a result of Minakshisundaram and Pleijel (1949, MR0031145 and 1953, MR0061750 ${ }^{2}$ that the heat trace admits an asymptotic expansion as $t$ approaches zero:

$$
\operatorname{Tr}\left(e^{-t \Delta_{g}}\right) \stackrel{t \searrow 0}{\sim}(4 \pi t)^{-n / 2} \sum_{k=0}^{\infty} a_{k}(M, g) t^{k},
$$

where $n$ is the dimension of $M$ and the coefficients $\left\{a_{k}(M, g)\right\}_{k=0}^{\infty}$, commonly referred to as the heat invariants of $(M, g)$, are integrals of universal polynomials $\left\{u_{k}(M, g)\right\}_{k=0}^{\infty}$ in the components of the Riemann curvature tensor and its covariant derivatives:

$$
a_{k}(M, g)=\int_{M} u_{k}(M, g) d v_{g}
$$

\footnotetext{
${ }^{2}$ The Notices of the AMS limits the bibliography to 20 references. Works not contained in the bibliography will be given an in-text citation of the form (Year of Publication, Mathematical Reviews or ArXiv Identifier).
}

Therefore, the asymptotics of the heat trace tell us that the spectrum encodes averages of certain local geometric data on the underlying manifold. Unfortunately, although useful in obtaining positive results (see section 3), explicit formulae exist only for the first few heat invariants and only $a_{0}$ and $a_{1}$ appear to have natural geometric interpretations. For instance, it is known that $a_{0}(M, g)=\int_{M} 1 d v_{g}$ is the volume of the Riemannian manifold and $a_{1}(M, g)=$ $\frac{1}{6} \int_{M}$ Scal $d v_{g}$ is a sixth of the total scalar curvature, while $a_{2}(M, g)$ can be expressed as follows:

$$
a_{2}(M, g)=\frac{1}{360} \int_{M} 2\left(|R|^{2}-|\operatorname{Ric}|^{2}\right)+5 \operatorname{Scal}^{2} d \nu_{g},
$$

where $|\cdot|$ is the norm on tensors induced by $g$. Therefore, equation (4) implies the spectrum encodes the dimension, volume, and total scalar curvature of the underlying Riemannian manifold. And, since scalar curvature equals Gaussian curvature in dimension two, the Gauss-Bonnet Theorem implies the Euler characteristic of a closed surface $(\Sigma, g)$ is encoded in its spectrum: $\chi(\Sigma)=\frac{6}{2 \pi} a_{1}(\Sigma, g)$.

In spite of this promising start, it is well known that the spectrum does not fully encode the geometry of its underlying Riemannian manifold. The first non-trivial isospectral pair is due to Milnor (1964, MR0162204). It consists of two 16-dimensional flat tori $\Gamma_{1} \backslash \mathbb{E}^{16}$ and $\Gamma_{2} \backslash \mathbb{E}^{16}$, where $\mathbb{E}^{16}$ denotes $\mathbb{R}^{16}$ equipped with a flat metric and $\Gamma_{1}, \Gamma_{2} \leq \mathbb{R}^{16}$ are a pair of self-dual full-rank lattices discovered by Witt and having the following properties: (1) they are not conjugate via the isometry group of $\mathbb{E}^{16}$, which ensures the corresponding flat tori are non-isometric (see Example 13), and (2) for any $\lambda>0, \Gamma_{1}$ and $\Gamma_{2}$ contain the same number of vectors of length at most $\lambda$, which ensures the associated flat tori have the same spectra. ${ }^{3}$ It would take two decades for the mathematics community to begin to realize Milnor's isospectral manifolds are an instance of a systematic procedure for producing isospectral pairs that utilizes symmetry and representation theory.

\section{Isospectral Manifolds Via Symmetry}

To understand how symmetry enters the picture, first, notice the group of diffeomorphisms $\operatorname{Diff}(M)$ of a smooth manifold $M$ has a natural action on $L^{2}\left(M, d \nu_{g}\right)$ given by $(\Phi \cdot f)(x)=f\left(\Phi^{-1}(x)\right)$ for any $\Phi \in \operatorname{Diff}(M)$ and $f \in$ $L^{2}\left(M, d \nu_{g}\right)$, where $\nu_{g}$ is the associated Riemannian measure. A diffeomorphism $\Phi$ is said to commute with the Laplace operator $\Delta_{g}$ if $\Phi \cdot\left(\Delta_{g} f\right)=\Delta_{g}(\Phi \cdot f)$ for any $f \in L^{2}\left(M, d \nu_{g}\right)$. It is not difficult to see that in the event $\Phi$ is actually an isometry of $(M, g)$, then $\Phi$ commutes with $\Delta_{g}$. In fact, it is a result of B. Watson (1973, MR0365419) that this

\footnotetext{
${ }^{3}$ One can show that the eigenvalue set of a flat torus $\Gamma \backslash \mathbb{E}^{n}$ is $\mathscr{E}_{\Delta}\left(\Gamma \backslash \mathbb{E}^{n}\right)=$ $\left\{4 \pi^{2}\left\|\gamma^{*}\right\|^{2}: \gamma^{*} \in \Gamma^{*}\right\}$, where $\Gamma^{*}$ is the dual lattice of $\Gamma$, and the multiplicity of $\lambda \in \mathscr{E}_{\Delta}\left(\Gamma \backslash \mathbb{E}^{n}\right)$ is equal to $\#\left\{\gamma^{*} \in \Gamma^{*}:\left\|\gamma^{*}\right\|^{2}=\frac{\lambda}{4 \pi^{2}}\right\}$.
} 
property characterizes the elements of $\operatorname{Diff}(M)$ that are isometries of $(M, g)$. Consequently, if $G$ is a Lie group consisting of isometries of $(M, g)$, then the $\Delta_{g}$-eigenspaces are representations of $G$ under its natural action on $L^{2}\left(M, d \nu_{g}\right)$.

Second, recall that an embedded submanifold $F$ of a Riemannian manifold $(M, g)$ is said to be totally geodesic if the geodesics in $F$ equipped with the metric induced by $g$ remain geodesics when viewed in the ambient space $(M, g)$. For example, since the geodesics in Euclidean $n$-space $\mathbb{E}^{n}$ are straight lines we see that any plane $\Pi \subset \mathbb{E}^{n}$ is a totally geodesic submanifold, while the round sphere $\mathbb{S}^{n-1} \subset \mathbb{E}^{n}$, whose geodesics are segments of great circles, is not a totally geodesic submanifold. A map $\pi:(M, g) \rightarrow(N, h)$ between two Riemannian manifolds is said to be a Riemannian submersion if $\pi$ is a submersion and for each $q \in N$ and $p \in F_{q} \equiv \pi^{-1}(q)$ we have $\pi_{*}:\left(\mathcal{H}_{p}, g_{p}\left\lceil\mathcal{H}_{p}\right) \rightarrow\left(T_{q} N, h_{q}\right)\right.$ is an isometry, where $T_{p} M=T_{p} F_{q} \oplus \mathcal{H}_{p}$ is an orthogonal direct sum. A Riemannian submersion $\pi:(M, g) \rightarrow(N, h)$ is said to have totally geodesic fibers if for any $q \in N$, $F_{q}=\pi^{-1}(q)$ is a totally geodesic submanifold of $(M, g)$. Under the assumption that $\pi:(M, g) \rightarrow(N, h)$ is a Riemannian submersion with totally geodesic fibers, it can be shown that for any $\lambda \in \mathbb{R}$ and $f \in C^{\infty}(N)$ we have $\Delta_{h} f=\lambda f$ if and only if $\Delta_{g}(f \circ \pi)=\lambda(f \circ \pi)$.

Putting the previous observations together, we find that if the Lie group $H$ acts freely via isometries on $(M, g)$ and with orbits that are totally geodesic submanifolds, then $\mathscr{E}_{\Delta}\left(H \backslash M, g_{H}\right) \subseteq \mathscr{E}_{\Delta}(M, g)$ and the multiplicity $m_{\Delta}(\lambda)$ of each $\lambda \in \mathscr{E}_{\Delta}\left(H \backslash M, g_{H}\right)$ is equal to the dimension of $L^{2}\left(M, v_{g_{H}}\right)_{\lambda}^{H}$, where $g_{H}$ is the quotient metric on $H \backslash M$ induced by $g$ and for any (unitary) representation $\rho: K \rightarrow$ $\mathrm{U}(V)$ we let $V^{K} \equiv\{v \in V: \rho(x) v=v$ for all $x \in K\}$.

2.1. A generalized Sunada method. Sunada's method and its generalizations are perhaps the most widely known of the symmetry techniques used to construct non-trivial isospectral manifolds. In Sunada's original formulation, the method exploits an algebraic condition (see equation (6)) on triples of finite groups that was already known to F. Gassmann in 1926, who was interested in constructing non-isomorphic number fields with identical Dedekind Zeta functions. For our exposition it will be convenient to present this algebraic condition in terms of the so-called "dimension datum" of a group and expand the groups under consideration to include compact Lie groups; this is different from the usual presentation of this material as is found in [Bu], for example.

Definition 1. Let $G$ be a compact Lie group, and let $\widehat{G}$ denote the equivalence classes of irreducible representations $\left(V_{\rho}, \rho\right)$ of $G$. Given a closed subgroup $H \leq G$, its dimension datum (relative to $G$ ) is the map $\mathscr{D}_{H}^{G}: \widehat{G} \rightarrow \mathbb{Z}$ defined by $\left[\left(V_{\rho}, \rho\right)\right] \mapsto \operatorname{dim} V_{\rho}^{H}$.
A triple of compact groups $\left(G, H_{1}, H_{2}\right)$ is said to be a Gassmann-Sunada triple or Sunada triple if (1) $H_{1}, H_{2} \leq G$ and (2) $\mathscr{D}_{H_{1}}^{G}=\mathscr{D}_{H_{2}}^{G}$. A Sunada triple $\left(G, H_{1}, H_{2}\right)$ is said to be non-trivial if $H_{1}$ and $H_{2}$ are not conjugate in $G$. In the event we have a Sunada triple $\left(G, H_{1}, H_{2}\right)$, where $G$ is finite, one can see that the condition $\mathscr{D}_{H_{1}}^{G}=\mathscr{D}_{H_{2}}^{G}$ is equivalent to the classical Gassmann-Sunada condition

$$
\#\left(H_{1} \cap g^{G}\right)=\#\left(H_{2} \cap g^{G}\right) \text { for all } g \in G,
$$

where $g^{G}$ denotes the conjugacy class of a group element $g$ within $G$.

Our previous observations regarding the interplay between isometries and the Laplace spectrum allow us to deduce the following generalization of Sunada's method.

Theorem 2 (Sutton [Sut]). Let $(M, g)$ be a compact Riemannian manifold and $\left(G, H_{1}, H_{2}\right)$ a Sunada triple, where $G \leq \operatorname{Isom}(M, g)$ and, for $j=1,2, H_{j}$ acts freely on $M$ such that the natural projection $\pi_{j}:(M, g) \rightarrow\left(H_{j} \backslash M, g_{j}\right)$ has totally geodesic fibers. Then, the Riemannian manifolds $\left(H_{1} \backslash M, g_{1}\right)$ and $\left(\mathrm{H}_{2} \backslash M, \mathrm{~g}_{2}\right)$ are isospectral.

We emphasize that Theorem 2 is silent on whether the resulting spaces are isometric; that matter must be handled separately. ${ }^{4}$

In Sunada's seminal paper [Su], the previous theorem is stated for a Sunada triple of finite groups; in which case, the requirement that the projection maps have totally geodesic fibers is always achieved and the resulting quotient spaces are necessarily locally isometric, since they will have a common Riemannian covering. When invoked with a Sunada triple of finite groups, we will refer to the method as the classical Sunada method (see Example 4). ${ }^{5}$ There are a number of ways of manufacturing Sunada triples of finite groups (see [DGS, sec. 7]), many of which predate Sunada's paper due to their connections with problems in group theory and number theory. We now review a procedure for constructing Sunada triples that disproves Littlewood's conjecture (1940, MR0002127) that subgroups of a finite group $G$ possessing the same dimension datum must be isomorphic.

Example 3 (Todd-Komatsu method). Let $H_{1}$ and $H_{2}$ be two finite groups of order $n$ such that, for any divisor $d$ of $n$, the groups $H_{1}$ and $H_{2}$ have the same number of elements of order $d$. By numbering the group elements and allowing them to act on themselves via left-multiplication, $H_{1}$ and $H_{2}$ can be embedded in $S_{n}$, the symmetric group on $n$ elements. Under this embedding, each element of order $d$ is sent to a product of $n / d$ disjoint cycles of length

\footnotetext{
${ }^{4}$ For other variants of Sunada's method we point the reader to the work of D. DeTurck and C. Gordon (1989, MR1029118) and H. Pesce (1996, MR1396675), for example.

${ }^{5}$ S. Chen (1992, MR1184007 showed that Milnor's isospectral tori mentioned in section 1 can be viewed as arising via the classical Sunada method.
} 
$d$. Recalling that two elements in $S_{n}$ are conjugate if and only if they have the same cycle decomposition, it follows that the elements of order $d$ in $H_{1}$ and $H_{2}$ are pairwise conjugate in $S_{n}$. Therefore, the triple $\left(S_{n}, H_{1}, H_{2}\right)$ is a Sunada triple and it is non-trivial precisely when $H_{1}$ and $H_{2}$ are not isomorphic. The first instance of this construction is found in the work of J. A. Todd (1950) in which the nontrivial Sunada triple $\left(S_{16}, H_{1}, H_{2}\right)$ is formed by $H_{1}=\mathbb{Z}_{8} \times \mathbb{Z}_{2}$ and $H_{2}=\mathbb{Z}_{8} \rtimes_{\varphi} \mathbb{Z}_{2}$, where $\varphi: \mathbb{Z}_{2} \rightarrow \operatorname{Aut}\left(\mathbb{Z}_{8}\right)$ is defined by $\varphi(\overline{1})(\bar{x})=\overline{x^{5}}$. Subsequently, K. Komatsu (1976) found a family of triples that also fit into this paradigm: let $p$ be an odd prime; then $\left(S_{p^{3}}, H_{1}, H_{2}\right)$, where $H_{1} \simeq \mathbb{Z}_{p}^{3}$ and $H_{2}$ is a non-abelian group of order $p^{3}$ and exponent $p$, is a non-trivial Sunada triple.

As we noted earlier, Sunada's method does not guarantee the resulting quotients will be non-isometric. In the case of finite groups, one may obtain non-isometric spaces by using nowhere locally homogeneous metrics. A metric $g$ on a manifold $M$ is said to be nowhere locally homogeneous if for any pair of distinct open sets $U$ and $V$ in $M$ there is no isometry between $(U, g \uparrow U)$ and $(V, g \uparrow V)$, where $\uparrow$ denotes restriction. Sunada showed that if $M$ is a compact manifold of dimension at least two, the nowhere locally homogeneous metrics on $M$ form a residual set in $\mathscr{R}(M)$, the space of Riemannian metrics on $M$ [Su, Prop. 1]. This observation can be combined with Sunada's method to produce examples of isospectral, yet non-isometric, manifolds.

Example 4 (The classical Sunada method). Fix a nontrivial Gassmann-Sunada triple $\left(G, H_{1}, H_{2}\right)$ of finite groups and let $M_{0}$ be a compact manifold such that there exists an epimorphism $\varphi: \pi_{1}\left(M_{0}\right) \rightarrow G$. (As every finitely generated group is the fundamental group of some closed four-manifold, this step is always possible.) Then, let $\pi: M \rightarrow M_{0}$ be the normal covering of $M_{0}$ corresponding to $\operatorname{ker}(\varphi) \triangleleft \pi_{1}\left(M_{0}\right)$. Next, endow $M_{0}$ with a nowhere locally homogeneous metric $g_{0}$ and let $g=\pi^{*} g_{0}$ be the corresponding Riemannian covering metric on $M$. Then, $\operatorname{Isom}(M, g)=G$ and the quotient manifolds $\left(H_{1} \backslash M, g_{1}\right)$ and $\left(H_{2} \backslash M, g_{2}\right)$ are isospectral manifolds that are not isometric. Indeed, in light of the fact that $\operatorname{Isom}(M, g)=G$, an isometry between $\left(H_{1} \backslash M, g_{1}\right)$ and $\left(H_{2} \backslash M, g_{2}\right)$ would imply that $H_{1}$ and $H_{2}$ are conjugate in $G$, a contradiction. We note that, in the event that $H_{1}$ and $H_{2}$ are non-isomorphic groups (e.g., Example 3), we can choose any $G$-invariant metric on $M$, since $H_{1} \backslash M$ and $H_{2} \backslash M$ are not homeomorphic.

Modern differential geometry has its origins in the study of surfaces. As such, there has been considerable interest in the existence of isospectral surfaces; especially, those of constant (negative) sectional curvature. Given a non-trivial Sunada triple $\left(G, H_{1}, H_{2}\right)$, one can construct

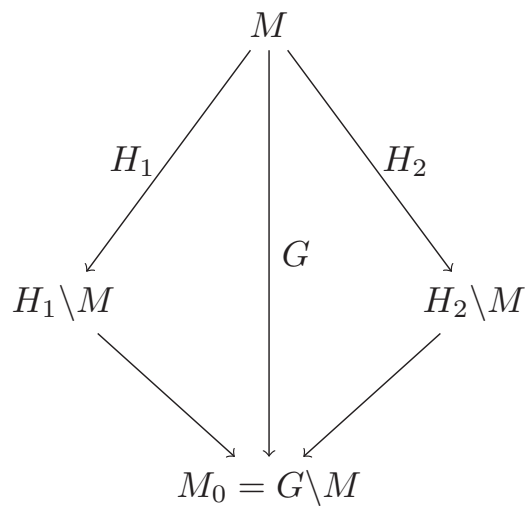

Figure 2. Diagram of covering spaces in the classical Sunada method (Example 4).

non-trivial pairs of isospectral surfaces (of constant negative sectional curvature) with the assistance of the Cayley graphs associated to $G, H_{1} \backslash G$, and $H_{2} \backslash G$. Rather than give the details of this construction, which is described beautifully by Buser [Bu], we pivot to discuss a somewhat surprising application of Sunada's method: the existence of simply-connected isospectral manifolds.

2.2. Isospectral simply-connected manifolds via Sunada's method. A long-standing problem in spectral geometry was to determine whether isospectral simplyconnected manifolds are necessarily isometric. This was partially motivated by the fact that it was a feature of the earliest construction techniques that the resulting isospectral manifolds have non-trivial fundamental groups. The generalized Sunada method given in Theorem 2 was introduced by the author [Sut] as a means to produce isospectral simply-connected Riemannian manifolds that are not even homeomorphic.

Given a connected compact Lie group $G$ and a closed subgroup $H \leq G$, the coset space $H \backslash G$ admits a unique smooth manifold structure of dimension $\operatorname{dim} G-\operatorname{dim} H$ with respect to which the natural projection map $\pi: G \rightarrow$ $H \backslash G$ is a submersion. For any $g \in G$, we let $L_{g}, R_{g}$ : $G \rightarrow G$ denote left and right multiplication by $g$, respectively. Then, a Riemannian metric $m$ on $G$ is said to be bi-invariant if $L_{g}$ and $R_{g}$ are isometries of $(G, m)$ for any $g \in G$, and such metrics always exist on a compact Lie group. Now, any bi-invariant metric $m$ on $G$ induces a Riemannian metric $m^{\prime}$ on $H \backslash G$ such that $\pi:(G, m) \rightarrow$ $\left(H \backslash G, m^{\prime}\right)$ is a Riemannian submersion with totally geodesic fibers. We can examine the topology of the quotient manifold $H \backslash G$ through the homotopy long exact sequence

$$
\begin{aligned}
\cdots \rightarrow \pi_{2}(G) & \rightarrow \pi_{2}(H \backslash G) \\
& \rightarrow \pi_{1}(H) \rightarrow \pi_{1}(G \backslash G) \\
& \rightarrow \pi_{0}(H) \rightarrow \cdots
\end{aligned}
$$

and find that, in the event that $G$ and $H$ are both connected and $G$ is simply-connected, the manifold $H \backslash G$ is 
simply-connected. To compare the geometry of two such quotient spaces, suppose $G$ is simple and $H_{1}, H_{2} \leq G$ are not conjugate via an automorphism of $G$ (i.e., there is no automorphism $\alpha \in \operatorname{Aut}(G)$ such that $\left.\alpha\left(H_{1}\right)=H_{2}\right)$. Then one can check that $\left(H_{1} \backslash G, m_{1}\right)$ and $\left(H_{2} \backslash G, m_{2}\right)$ are not isometric, where $m_{1}$ and $m_{2}$ are the quotient metrics induced by a common bi-invariant metric $m$ on $G$. Hence, to construct the desired example, it is sufficient to find a Sunada triple $\left(G, H_{1}, H_{2}\right)$ of connected Lie groups where $G$ is simple and $H_{1}$ and $H_{2}$ are not conjugate via $\operatorname{Aut}(G)$.

Examining the work of Larsen and Pink on dimension datum [LP], one notices that there is a connected semisimple Lie group $H$ such that for infinitely many $n \in \mathbb{N}$ there are faithful representations $\rho_{1}, \rho_{2}: H \rightarrow \mathrm{SU}(n)$, such that $H_{1}=\rho_{1}(H)$ and $H_{2}=\rho_{2}(H)$ are not conjugate via $\operatorname{Aut}(\mathrm{SU}(n))$ and $\mathscr{D}_{H_{1}}^{\mathrm{SU}(n)}=\mathscr{D}_{\mathrm{H}_{2}}^{\mathrm{SU}(n)}$. Therefore, $\left(\mathrm{SU}(n), H_{1}, H_{2}\right)$ is a suitable Sunada triple.

Theorem 5 (Sutton [Sut]). There exist non-trivial Sunadaisospectral pairs of simply-connected Riemannian manifolds.

These examples were published shortly after Schueth [Sc] and Gordon [Go2] each constructed their own examples of simply-connected isospectral manifolds via what is sometimes referred to as the "torus method" (see section 2.3). It is clear that the underlying spaces in the examples of Schueth and Gordon are homeomorphic; however, as we noted earlier, the motivation behind our generalized Sunada technique was to produce non-homeomorphic simply-connected isospectral pairs. Unfortunately, the abstract nature of the triple (SU $\left.(n), H_{1}, H_{2}\right)$ employed in [Sut] and the dimension of the smallest such examples (approximately $10^{10}$ ) proved to be a barrier at the time. However, through a more detailed analysis of dimension datum, An, Yu, and Yu were able to exhibit Sunada triples of connected Lie groups, as above, where the resulting isospectral simply-connected quotient spaces are nonhomeomorphic [AYY].

Specifically, for $n \geq 2$, let $H_{1}=U(n)$ and $H_{2}=$ $\operatorname{Sp}\left(n_{1}\right) \times \operatorname{SO}\left(2 n-2 n_{1}\right)$, where $n_{1}=\left\lfloor\frac{n-1}{2}\right\rfloor$. And, let $\mathrm{st}_{1}, \mathrm{st}_{2}$, and $\mathrm{st}_{3}$ denote the standard representations of $H_{1}, \operatorname{Sp}\left(n_{1}\right)$, and $\operatorname{SO}\left(2 n-2 n_{1}\right)$, respectively. Then, $H_{1}$ and $H_{2}$ can be embedded into $\mathrm{SU}(2 n)$ via $\mathrm{st}_{1} \oplus \mathrm{st}_{1}^{*}$ and $\left(s t_{2} \otimes \mathbf{1}\right) \oplus\left(\mathbf{1} \otimes s_{3}\right)$, respectively. Under this identification they show that $\left(\mathrm{SU}(2 n), H_{1}, H_{2}\right)$ is a non-trivial Sunada triple, where $H_{1}$ and $H_{2}$ are not isomorphic (cf. Example 3) and, since $\mathbb{Z} \simeq \pi_{1}\left(H_{1}\right) \nsim \pi_{1}\left(H_{2}\right) \simeq$ $\mathbb{Z}_{2}$, the long exact sequence (see equation (7)) allows us to conclude that the quotient spaces $H_{1} \backslash \mathrm{SU}(2 n)$ and $H_{2} \backslash \mathrm{SU}(2 n)$ are not homeomorphic. Therefore, by Theorem $2,\left(H_{1} \backslash \mathrm{SU}(2 n), m_{1}\right)$ and $\left(H_{2} \backslash \mathrm{SU}(2 n), m_{2}\right)$ are nonhomeomorphic simply-connected isospectral manifolds, where $m_{1}$ and $m_{2}$ are induced by a bi-invariant metric $m$ on $\mathrm{SU}(2 n)$. And we conclude that the topological universal cover of a Riemannian manifold is not a spectral invariant.

Theorem 6 (An, Yu, and $\mathrm{Yu}[\mathrm{AYY}])$. There are infinitely many pairs of simply-connected Sunada-isospectral manifolds that are not homeomorphic.

Although not mentioned in the article, these examples also show that, among homogeneous spaces, model geometries are not spectral invariants, in general (see Problem 3).

In light of the preceding discussion, it is natural to wonder whether there are additional non-trivial Sunada triples $\left(G, H_{1}, H_{2}\right)$ consisting of connected compact Lie groups. In his engaging doctoral thesis, Jun $\mathrm{Yu}$-the third of the aforementioned authors-classifies the connected closed subgroups of a given compact Lie group with identical dimension datum [Yu]. Although of interest because of its connection to the inverse spectral problem, Yu's classification theorem appears to have been primarily motivated by Langland's suggestion that dimension datum will play a key role in his "Beyond Endoscopy" program [Yu, p. 2684].

2.3. The Gordon-Schueth method. While its connections with problems in group theory, number theory, and geometry make Sunada's method the most well-known technique for producing isospectral manifolds, another effective approach exploits isometric actions by tori. Over the last three decades this technique has been developed and refined primarily by Carolyn Gordon and Dorothee Schueth, with initial inspiration drawn from an earlier approach of Szabo, ${ }^{6}$ and it is responsible for a number of notable firsts in the spectral geometry canon: (1) isospectral closed manifolds with distinct local geometries [Go1], (2) non-trivial isospectral deformations [GoWi], and (3) pairs of non-trivial isospectral metrics on a simply-connected manifold [Sc, Go2].

Given a Riemannian manifold $(M, g)$ and an isometric free action by a torus $T$, let $g^{T}$ denote the Riemannian metric on the quotient space $T \backslash M$ such that the natural projection $\pi:(M, g) \rightarrow\left(T \backslash M, g^{T}\right)$ is a Riemannian submersion. For each irreducible representation $\rho: T \rightarrow$ Aut $(\mathbb{C})$ of $T$, let $\mathscr{H}_{\rho}$ be the subspace of $L^{2}(M)$ defined by $\mathscr{H}_{\rho}=\left\{f \in L^{2}(M): f\left(g^{-1} \cdot x\right)=\rho(g)(f(x))\right.$ for all $\left.g \in T\right\}$. Then, we have the following decomposition of $L^{2}(M)$ :

$$
L^{2}(M)=\mathscr{H}_{0} \oplus\left(\bigoplus_{W \leq T}\left(\bigoplus_{\substack{\rho \in \hat{T} \\ \operatorname{ker}(\rho)=W}} \mathscr{H}_{\rho}\right)\right),
$$

${ }^{6}$ In 1992, Z. I. Szabó circulated a preprint in which he constructed the first pair of isospectral manifolds with boundary having different local geometries. These examples were published in 1999 as [Sz]. 
where the sum is taken over closed subgroups $W \leq T$ of codimension one. Now, keeping in mind our previous discussion of the behavior of the spectrum under Riemannian submersions with totally geodesic fibers, one can deduce the following criteria for isospectrality, which is sometimes referred to as the "torus method."

Theorem 7 (Gordon-Schueth method [Sc]). Let $\left(M_{1}, g_{1}\right)$ and $\left(M_{2}, g_{2}\right)$ be closed Riemannian manifolds, and, for $j=$ 1,2 , let $\alpha_{j}: T \times M_{j} \rightarrow M_{j}$ be a free isometric action by a $k$-dimensional torus $T$. Now, suppose that for any $W \leq T$ a closed subgroup that is either $T$ or a subtorus of codimension 1 the following hold:

1. For $j=1,2$ the fibers of the projection $\pi_{j}: M_{j} \rightarrow W \backslash M_{j}$ are totally geodesic in $\left(M_{j}, g_{j}\right)$.

2. $\left(W \backslash M_{1}, g_{1}^{W}\right)$ and $\left(W \backslash M_{2}, g_{2}^{W}\right)$ are isospectral.

Then, $\left(M_{1}, g_{1}\right)$ and $\left(M_{2}, g_{2}\right)$ are isospectral.

As is the case with Sunada's method, the proof of the Gordon-Schueth method is fairly straightforward. The art is in finding non-trivial applications.

The Gordon-Schueth method made its debut in the work of Gordon [Go1], where it was stated for the special case of nilmanifolds and was applied to produce the first examples of isospectral closed manifolds with different local geometries: the metrics are locally homogeneous metrics on an eight-dimensional nilmanifold. Subsequently, Gordon and Wilson used this technique to construct the first continuous families of isospectral metrics on manifolds with boundary [GoWi]. In 1999, Schueth provided the more general version of the method stated above and applied it to construct the first examples of isospectral metrics with different local geometries on a simply-connected space.

Theorem 8 (Schueth [Sc]). Let $G$ be a compact simplyconnected Lie group of rank at least two (e.g., $\mathrm{SU}(2) \times \mathrm{SU}(2))$ and $S^{n}$ the $n$-sphere. Then, there exists a continuous family $g_{t}$ of isospectral, yet non-isometric, metrics on $S^{n} \times G$.

Using a variant of Theorem 7 that only insists the torus action be free on the principal orbits, while placing a stronger requirement on the relationship between the quotient spaces $\left(W \backslash M_{1}, g_{1}^{T}\right)$ and $\left(W \backslash M_{2}, g_{2}^{T}\right)$, Gordon constructed continuous families of isospectral metrics on $S^{n}$ $(n \geq 8)$.

Theorem 9 (Gordon [Go2]). There exists a continuous family $g_{t}$ of isospectral, yet non-isometric, metrics on $S^{n}$.

The isospectral families of Theorem 8 (respectively, Theorem 9) can be made to come arbitrarily close to a given symmetric metric (see section 3) on $S^{n} \times G$ (respectively, $\left.S^{n}\right)$.

\section{Can One Hear the Geometry of a Riemannian Manifold with a "Large" Symmetry Group?}

If we measure the complexity of a Riemannian manifold in terms of sectional curvature, we obtain the following natural classes of spaces presented in order of increasing complexity:

- a Riemannian manifold $(M, g)$ is said to be a space of constant sectional curvature if there is a scalar $K$ such that $\operatorname{Sec}(\sigma)=K$ for all two-dimensional subspaces $\sigma \leq$ $T_{p} M, p \in M$;

- a Riemannian manifold $(M, g)$ is said to be locally symmetric if sectional curvature is constant under parallel transport, ${ }^{7}$ and if, in addition, $(M, g)$ is homogeneous (see below), then we say $(M, g)$ is (globally) symmetric;

- a Riemannian manifold $(M, g)$ is said to be locally homogeneous if the geometry in the neighborhood of any two points is identical; that is, any two points $p, q \in M$ have neighborhoods $U$ and $V$, respectively, where $(U, g\lceil U)$ and $(V, g \uparrow V)$ are isometric. The space $(M, g)$ is said to be (globally) homogeneous if its isometry group acts transitively on $M$.

The notion of a Riemannian manifold with "large" symmetry group can be formalized in several natural ways; however, for our discussion, homogeneous spaces and, more generally, locally homogeneous spaces will be considered spaces with "large" symmetry groups.

In dimension two the aforementioned classes are identical, while in dimension three and higher the containments are proper. Although these spaces account for a mere slice of the universe of Riemannian manifolds, they are ubiquitous, in part, because they arise organically as model spaces and solutions to natural variational problems in geometry, physics, and other disciplines. Consequently, it is natural to consider the degree to which these classes and certain representatives thereof are distinguished via their spectra.

\footnotetext{
${ }^{7}$ Given a Riemannian manifold $(M, g)$ and a smooth curve $c(t)$ with $c(0)=p$, parallel transport along $c$ is the linear transformation $P_{t}: T_{p} M \rightarrow T_{c(t)} M$ such that $t \mapsto P_{t}(\mathbf{v})$ moves $\mathbf{v} \in T_{p} M$ along $c(t)$ in a manner that preserves the magnitude and "direction" of $\mathbf{v}$. For example, in Euclidean space, $P_{t}(\mathbf{v})=$ $(c(t), v)$ for any $\mathbf{v}=(p, v) \in T_{p} \mathbb{E}^{n}$ and smooth curve $c(t)$ with $c(0)=p$. On the round two-sphere $\mathbb{S}^{2}=\left\{x \in \mathbb{R}^{3}:\|x\|=1\right\}$, the parallel transport along the geodesic $\gamma_{\theta}(t)=(\cos \theta \sin t, \sin \theta \sin t, \cos t)$, where $\theta \in \mathbb{R}$ is fixed, is given by $P_{t}^{\theta}\left(c_{1} v_{1}+c_{2} v_{2}\right)=c_{1}\left(N_{\gamma_{\theta}(t)} \times \dot{\gamma_{\theta}}(t)\right)+c_{2} \dot{\gamma_{\theta}}(t)$, where $v_{1}=(p ;-\sin \theta, \cos \theta, 0), v_{2}=\dot{\gamma_{\theta}}(0) \in T_{p} \mathbb{S}^{2}, c_{1}, c_{2} \in \mathbb{R}$, and $N$ is the outwardly pointing unit normal vector field on $\mathbb{S}^{2}$. Hence, a Riemannian manifold $(M, g)$ is locally symmetric if for any smooth curve $c(t)$ and any twodimensional subspace $\sigma \leq T_{c(0)} M$ we have $\operatorname{Sec}\left(P_{t}(\sigma)\right)=\operatorname{Sec}(\sigma)$.

Equivalently, a Riemannian manifold $(M, g)$ is said to be locally symmetric if for any $p \in M$ there is an $r>0$ and isometry $\sigma_{p}$ of the open ball $B(p ; r)$ such that $\sigma_{p}(p)=p$ and $d\left(\sigma_{p}\right)_{p}=-\mathrm{Id}$. The map $\sigma_{p}$ is called the geodesic symmetry at $p$ as it reverses the direction of the geodesics passing through $p .(M, g)$ is globally symmetric if every geodesic symmetry extends to an isometry of the entire manifold.
} 
Problem 2. To what extent is the property of being of constant sectional curvature, locally symmetric, or, more generally, locally homogeneous encoded in the spectrum? Are some of these spaces uniquely characterized by their spectra?

There are several natural ways in which a Riemannian manifold can be distinguished by its spectrum:

- $(M, g)$ is said to be spectrally determined if for any Riemannian manifold $(N, h)$ such that $\operatorname{Spec}_{\Delta}(N, h)=$ $\operatorname{Spec}_{\Delta}(M, g)$ we have $(N, h) \simeq(M, g)$, where we interpret $\simeq$ as meaning "is isometric to";

- a metric $g_{0} \in \mathscr{R}(M)$ is said to be spectrally isolated if there is a neighborhood $\mathcal{U}$ of $g_{0}$ such that for any $g \in$ $\mathcal{u}$ with $\operatorname{Spec}_{\Delta}(M, g)=\operatorname{Spec}_{\Delta}\left(M, g_{0}\right)$ we have $g \simeq g_{0}$;

- a metric $g_{0} \in \mathscr{R}(M)$ is said to be spectrally rigid if for any smooth deformation $g_{t},-\epsilon<t<\epsilon$, of $g_{0}$ in $\mathscr{R}(M)$ such that $\operatorname{Spec}_{\Delta}\left(M, g_{t}\right)=\operatorname{Spec}_{\Delta}\left(M, g_{0}\right)$ for all $t$, then we have $g_{t} \simeq g_{0}$ for all $t$.

Our discussion of Problem 2 will primarily focus on the degree to which locally homogeneous spaces are spectrally determined up to universal Riemannian cover; that is, the degree to which the local geometry of a locally homogeneous space is audible.

It is known that, for each $K \in \mathbb{R}$ and $n=2, \ldots, 5$, a closed $n$-dimensional Riemannian manifold of constant sectional curvature $K$ is spectrally determined up to universal Riemannian cover.

Theorem 10 (Berger, Tanno [T1]). Let $(M, g)$ and $(N, h)$ be two isospectral closed Riemannian manifolds of dimension $2 \leq n \leq 5$ and fix $K \in \mathbb{R}$. Then, $(M, g)$ has constant sectional curvature $K$ if and only if $(N, h)$ has constant sectional curvature $K$.

Furthermore, the existence of various low-dimensional isospectral spaces of constant sectional curvature shows this is the optimal general statement regarding constant sectional curvature. The two-dimensional case of the preceding theorem was proven by M. Berger (1968, MR0239535) and established in dimension three, four, and five by S. Tanno in 1973 [T1]. Both arguments employ the heat invariants, which typically requires a fair degree of finesse. However, the case of surfaces is rather elementary as we demonstrate next.

Since, for any point in a surface $\Sigma$ the associated tangent space is two-dimensional, sectional curvature can be viewed as a smooth function on $\Sigma$; in fact, it is just Gaussian curvature. Therefore, letting $x$ be a real number, we may integrate the expression $(x-\operatorname{Sec})^{2}$ over $\Sigma$ to obtain the following degree two polynomial:

$$
\begin{aligned}
P_{(\Sigma, g)}(x) & \equiv \int_{\Sigma}(x-\mathrm{Sec})^{2} \\
& =a_{0}(\Sigma, g) x^{2}-6 a_{1}(\Sigma, g) x+18 a_{2}(\Sigma, g) .
\end{aligned}
$$

Hence, $(\Sigma, g)$ is a closed surface of constant sectional curvature $K$ if and only if $K$ is a root of the polynomial $P_{(\Sigma, g)}(x)$. Berger's result is now easily deduced from the fact that isospectral surfaces share the same heat invariants. This is particularly interesting in light of the Uniformization Theorem which states that every orientable closed surface $\Sigma$ admits a metric of constant sectional curvature, the sign of which is determined by the genus of the surface.

In summary, metrics of constant sectional curvature are intimately related with the topology of surfaces via the Uniformization Theorem and this intimacy is reflected in the spectrum by Berger's result (see Figure 3). To extend our exploration of Problem 2 to higher dimensions it will be convenient to frame our discussion in terms of geometries.

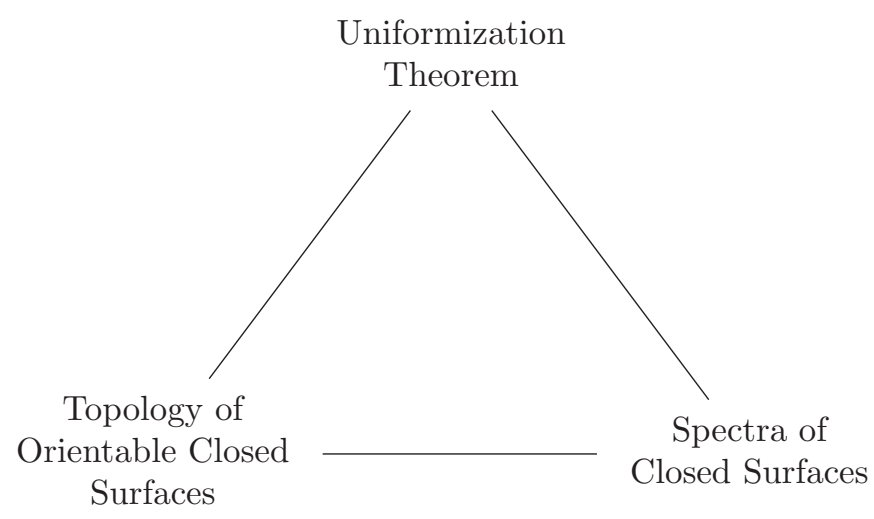

Figure 3. In dimension two there is an intimate relationship between the topology of closed orientable surfaces, metrics of constant sectional curvature, and their spectra.

3.1. Geometries, geometric structures, and the Laplace spectrum. Intuitively, an $n$-dimensional geometry is an ordered triple $(X, G, \alpha)$ that represents an infinite family of $n$-dimensional fabrics with highly symmetric embroidery, where (1) the patterns in the family share a "large" group of common symmetries and (2) for each fabric in the family there are certain compact $n$-dimensional Riemannian manifolds that are crafted from this material. A Riemannian manifold is said to be modeled on the geometry $(X, G, \alpha)$ if it is constructed from a fabric in this family. And, in each dimension, there is a collection of distinct geometries upon which all compact locally homogeneous Riemannian manifolds are modeled. While each embroidered fabric in a geometry $(X, G, \alpha)$ is highly symmetric, we are particularly interested in the patterns within the family that have a maximal amount of common symmetries. Such a subfamily constitutes what is known as a symmetry maximal geometry and consists of the most refined and preferred embroidered fabrics in the family: these are the "best" highly symmetric embroidered fabrics.

In precise mathematical terms, a geometry is an ordered triple $(X, G, \alpha)$, where $X$ is a connected and 
simply-connected manifold, $G$ is a connected Lie group, and $\alpha: G \times X \rightarrow X$ is a smooth transitive $G$-action on $X$ via diffeomorphisms with compact point stabilizers (i.e., for any $p \in M$ the group $G_{p} \equiv\{g \in G: g \cdot p \equiv \alpha(g, p)=p\}$ is compact) and there is a subgroup $\Gamma \leq G$ (acting freely and properly discontinuously) so that the quotient manifold $\Gamma \backslash X$ is compact. The condition that the $G$-action have compact point stabilizers implies $\mathscr{R}_{G}(X)$, the collection of $G$-invariant Riemannian metrics on $X$, is non-empty. ${ }^{8}$ And, we note that the Riemannian manifolds $(X, g)$, with $g \in \mathscr{R}_{G}(X)$, are the "embroidered fabrics" referred to in the metaphor above. When the risk of confusion is minimal we will abbreviate a geometry $(X, G, \alpha)$ as $(X, G)$.

Two geometries $\left(X_{1}, G_{1}, \alpha_{1}\right)$ and $\left(X_{2}, G_{2}, \alpha_{2}\right)$ are said to be equivalent if there exist a Lie group isomorphism $\Psi$ : $G_{1} \rightarrow G_{2}$ and a diffeomorphism $F: X_{1} \rightarrow X_{2}$ such that $F\left(\alpha_{1}(g, x)\right)=\alpha_{2}(\Psi(g), F(x))$. A geometry $(X, H, \beta)$ is called a subgeometry of $(X, G, \alpha)$, denoted by $(X, H, \beta) \leq(X, G, \alpha)$, if $H \leq G$ and $\alpha$ restricted to $X \times H$ agrees with $\beta$, in which case we conclude $\mathscr{R}_{G}(X) \subseteq \mathscr{R}_{H}(X)$. And, a geometry is said to be symmetry maximal ${ }^{9}$ if (up to equivalence) it is not a proper subgeometry of another geometry. ${ }^{10}$ Therefore, if $(X, G)$ is a symmetry maximal geometry, we have $\operatorname{Isom}(X, g)^{o}=G$ for any $g \in \mathscr{R}_{G}(X)$, and there is no metric on $X$ that enlarges this symmetry group. In our metaphor, the simply-connected Riemannian manifolds $(X, g)$ with $g \in \mathscr{R}_{G}(X)$ corresponding to a fixed symmetry maximal geometry $(X, G)$ form a minimal family consisting of the best highly symmetric embroidered fabrics (see Figure 4 ).

Complementary to the notion of a symmetry maximal geometry, we will say that a geometry $(X, G)$ is metrically maximal if whenever $(X, H) \leq(X, G) \lesseqgtr(X, L)$, we have $\mathscr{R}_{L}(X) \subsetneq \mathscr{R}_{G}(X)=\mathscr{R}_{H}(X)$. In this case, among the homogeneous Riemannian metrics on $X$ that admit compact quotients, $\mathscr{R}_{G}(X)$ is a maximal collection of metrics (possessing a common connected group of symmetries) and $G$ is minimal among $\operatorname{Isom}(X, h)^{o}$ as $h$ ranges over homogeneous metrics on $X$ admitting compact quotients. Therefore, up to isometry, the collection of homogeneous metrics on $X$ covering a compact quotient (abbreviated c.q.) is precisely

$$
\mathscr{R}_{\text {hom }}^{\mathrm{cq}}(X) \equiv \bigcup_{[(X, G)]} \mathscr{R}_{G}(X),
$$

where the union is taken over all equivalence classes of

\footnotetext{
${ }^{8}$ Since $G$ acts transitively on $X$, the metrics in $\mathscr{R}_{G}(X)$ are homogeneous and, therefore, $G$ is a "large" group of common symmetries for the metrics in $\mathscr{R}_{G}(X)$.

${ }^{9}$ This is typically referred to simply as "maximal"; however, we have settled on "symmetry maximal" because it is a bit more descriptive and is more easily distinguished from our new notion of "metrically maximal," which we introduce in the next paragraph.

${ }^{10}$ It is known that every geometry is a subgeometry of some symmetry maximal geometry and up through dimension four it is unique. Interestingly, A. Geng (2016, MR3553586) observed that in dimension five there is a geometry contained in two distinct symmetry maximal geometries.
}

\author{
n-dimensional \\ "Embroidered Fabric"
}

$$
\underbrace{\left\{(X, g): g \in \mathscr{R}_{G_{S M}}(X)\right\}}_{\text {Symmetry Maximal }} \subseteq \underbrace{\left\{(X, g): g \in \mathscr{R}_{G_{M M}}(X)\right\}}_{\text {Metrically Maximal }}
$$

Figure 4. Let $\left(X, G_{M M}\right)$ be a metrically maximal subgeometry of the $n$-dimensional symmetry maximal geometry $\left(X, G_{S M}\right)$. The diagram shows the relationship between the $n$-dimensional simply-connected Riemannian manifolds (or "embroidered fabrics") associated to these geometries. The Riemannian manifolds corresponding to the symmetry maximal geometry are considered to be "the best" or "preferred."

metrically maximal geometries on $X$. In our metaphor, the simply-connected Riemannian manifolds $(X, g)$ with $g \in \mathscr{R}_{G}(X)$ corresponding to a fixed metrically maximal geometry $(X, G)$ form a maximal family of highly symmetric embroidered fabrics (see Figure 4).

Example 11 (Constant curvature geometries). Let $\mathbb{E}^{n}$ denote $n$-dimensional Euclidean space, $\mathbb{S}^{n}$ the $n$-dimensional sphere with the round metric of constant sectional curvature +1 , and $\mathbb{\boxplus}^{n}$ hyperbolic $n$-space of constant sectional curvature -1 . Then we have the following symmetry maximal geometries:

- $\left(\mathbb{R}^{n}, \operatorname{Isom}\left(\mathbb{E}^{n}\right)^{o}=\mathbb{R}^{n} \rtimes \mathrm{SO}(n)\right)$, where (up to isometry) $\mathscr{R}_{\operatorname{Isom}\left(\mathbb{E}^{n}\right)^{o}}\left(\mathbb{R}^{n}\right)$ consists of the unique flat metric on $\mathbb{R}^{n}$;

- $\left(S^{n}, \operatorname{Isom}\left(\mathbb{S}^{n}\right)^{o}=\mathrm{SO}(n)\right)$, where (up to isometry) $\mathscr{R}_{\text {Isom }\left(\mathbb{S}^{n}\right)^{o}}\left(S^{n}\right)$ consists of the metrics of constant positive sectional curvature on $S^{n}$;

- $\left(H^{n}, \operatorname{Isom}\left(\mathbb{H}^{n}\right)^{o}\right)$, where (up to isometry) $\mathscr{R}_{\operatorname{Isom}\left(\uplus^{n}\right)^{o}}\left(H^{n}\right)$ consists of the metrics of constant negative sectional curvature on the upper half-space $H^{n}$.

That these geometries are symmetry maximal follows from the fact that, for any complete Riemannian $n$-manifold $(M, g), \operatorname{dim} \operatorname{Isom}(M, g) \leq \frac{n(n+1)}{2}$ with equality if and only if the space has constant sectional curvature and is either a simply-connected space or diffeomorphic to real projective space $\mathbb{R} P^{n}$. In dimension two, these are the only symmetry maximal geometries and these geometries are metrically maximal.

Example 12 (Symmetry maximal vs. metrically maximal). Let $H$ denote the quaternions. Then, the threesphere can be viewed as the space of unit quaternions $S^{3}=\{q \in H: q \cdot \bar{q}=1\}$, a group under multiplication. Now, for any $q \in H$, let $L_{q}$ and $R_{q}$ denote left and right multiplication, respectively. Then, for any $q \in S^{3}$, $L_{q}$ and $R_{q}$ are isometries of $S^{3}$ equipped with a standard "round metric." This leads to the universal Lie group 


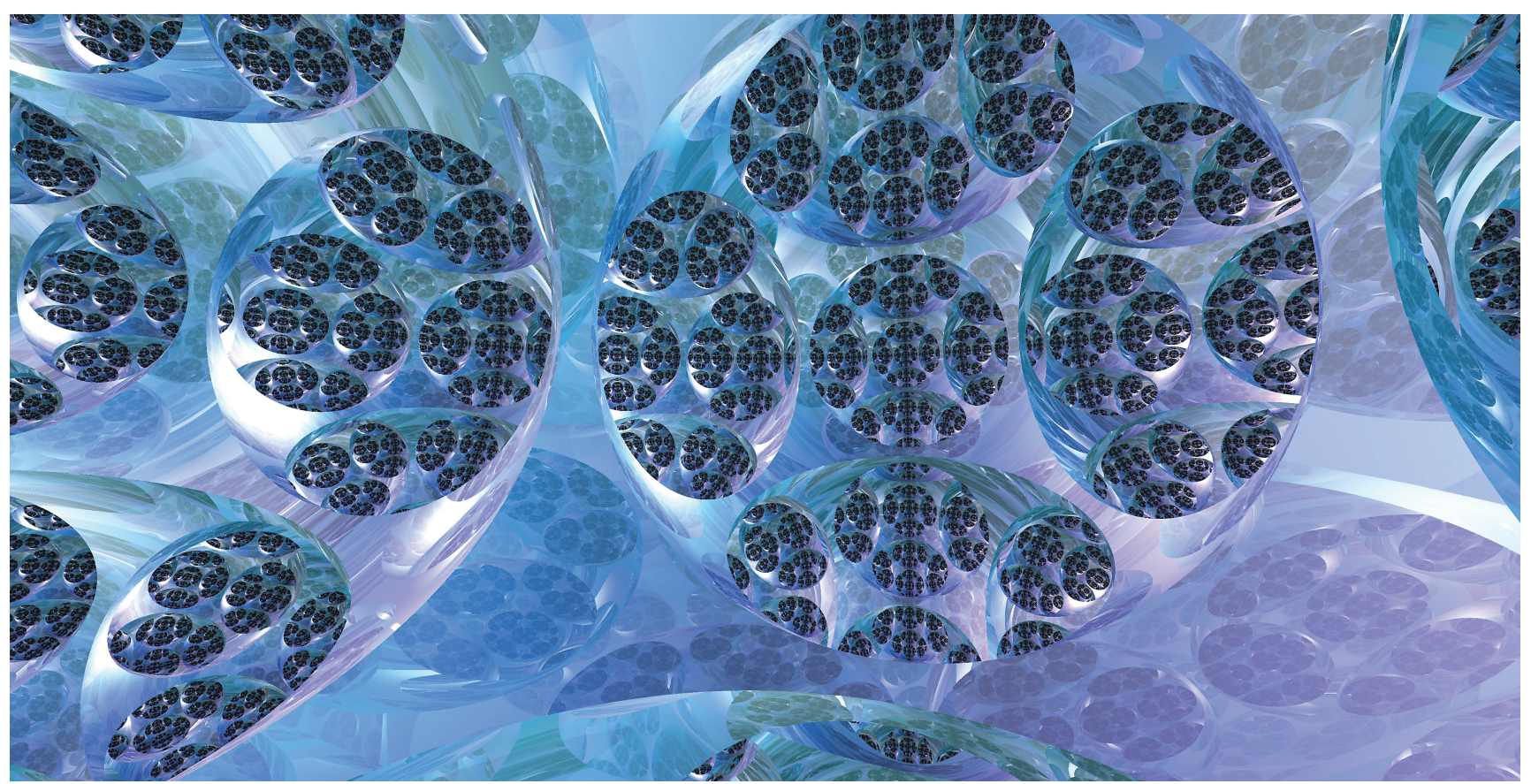

Figure 5. A cubical tiling of hyperbolic three-space $\mathbb{\boxplus}^{3}$, one of the eight Thurston geometries (see Theorem 14). Spaces modeled on $\mathbb{\boxplus}^{3}$ are determined up to local isometry by their spectra (see Theorem 10).

covering $\rho: S^{3} \times S^{3} \rightarrow \mathrm{SO}(4)$ given by $(g, h) \stackrel{\rho}{\mapsto} L_{g} \circ R_{h}$ and having kernel $\{ \pm(1,1)\}$. Then one can see:

- $\left(S^{3}, \mathrm{SO}(4)\right)$ is a symmetry maximal geometry and $\mathscr{R}_{\mathrm{SO}(4)}\left(S^{3}\right)$ consists of the metrics of constant sectional curvature on $S^{3}$ (see Example 11); and

- $\left(S^{3}, S^{3} \equiv \rho\left(S^{3} \times 1\right)\right)$ is a subgeometry of $\left(S^{3}, \mathrm{SO}(4)\right)$ that is metrically maximal. The associated space of invariant metrics $\mathscr{R}_{S^{3}}\left(S^{3}\right)$ is the collection of left-invariant metrics on $S^{3}$ and (up to isometry) it is a threedimensional space that accounts for all the homogeneous metrics on $S^{3}$.

[LSS1] contains a complete discussion of the symmetry maximal and metrically maximal geometries on standard spheres. ${ }^{11}$

Finally, a complete locally homogeneous Riemannian metric $g$ on a smooth manifold $M$ is called a geometric structure and it is said to be modeled on a geometry $(X, G)$ if its universal Riemannian cover is isometric to $X$ equipped with a $G$-invariant metric $\tilde{g}$, in which case we also say the metric $\tilde{g}$ induces the geometric structure $g$ on $M$. And, by a result of I. Singer (1960, MR0131248), every $n$ dimensional compact locally homogeneous Riemannian

\footnotetext{
${ }^{11}$ In dimension two and three it can be seen that (1) every metrically maximal geometry is a subgeometry of a unique symmetry maximal geometry and (2) every symmetry maximal geometry has a unique metrically maximal subgeometry. However, these statements are not true in general. Statement (1) fails in dimension five (see footnote 10) and, using Ziller's classification of homogeneous metrics on spheres (1982, MR0661203), one can see statement (2) is false in dimension seven.
}

manifold is modeled on some $n$-dimensional (metrically maximal) geometry. As we indicated earlier, we can consider the geometric structure $g$ as a realization of $M$ in the "embroidered fabric" $(X, \tilde{g})$. The next example shows a fixed embroidered fabric might actually give multiple geometrically distinct realizations of a given smooth manifold.

Example 13 (Flat structures on tori). The (symmetry maximal) geometry $\left(\mathbb{R}^{n}\right.$, Isom $\left.\left(\mathbb{E}^{n}\right)^{o}\right)$ accounts for the geometric structures of constant sectional curvature zero; i.e., the flat metrics on an $n$-manifold $M$. And, although there is a unique flat metric on $\mathbb{R}^{n}$ for $n \geq 2$, the $n$-torus admits a multidimensional family of flat geometric structures, up to isometry and scaling. Indeed, let $\Gamma_{1}, \Gamma_{2} \leq \mathbb{R}^{n}$ be full-rank lattices. Then, the flat tori $\Gamma_{1} \backslash \mathbb{E}^{n}$ and $\Gamma_{2} \backslash \mathbb{E}^{n}$ are diffeomorphic, yet isometric if and only if $\Gamma_{1}$ and $\Gamma_{2}$ are related via an orthogonal transformation of $\mathbb{E}^{n} .^{12}$

With the preceding discussion in mind, we proposed the following variation of Problem 2 in [LSS1].

Problem 3. Determine the degree to which the geometry of an $n$-dimensional geometric structure is encoded in its spectrum. Specifically, let $(M, g)$ be a compact locally homogeneous $n$-manifold modeled on the geometry $(X, G)$. Now, suppose $(N, h)$ is a compact Riemannian manifold that shares the same spectrum as $(M, g)$. Can we conclude that ...

\footnotetext{
${ }^{12}$ In [LSS2] we classify the geometric structures on elliptic three-manifolds.
} 
(a) $\ldots(N, h)$ is a locally homogeneous Riemannian manifold?

(b) $\ldots(N, h)$ is modeled on $(X, G)$ ?

(c) $\ldots(M, g)$ and $(N, h)$ have isometric universal Riemannian coverings?

(d) $\ldots(M, g)$ and $(N, h)$ are isometric?

To what extent do the answers to these questions depend on the specific choice of geometry $(X, G)$ or whether the geometry is symmetry maximal?

Each of these questions has a negative answer in sufficiently large dimension. For instance, Theorem 6 shows that in dimension 26 and higher the answer to Problem 3(b) is negative. However, with the exception of part (d), they are unresolved in dimension three. Moreover, the positive resolution of the Geometrization Conjecture shows that in dimension three there is an intimate relationship between the topology of closed three-manifolds and locally homogeneous three-manifolds; specifically, compact manifolds modeled on the so-called Thurston geometries (see Theorem 14) serve as the "building blocks" for closed three-manifolds: every oriented prime closed threemanifold has a canonical decomposition into pieces each of which admits geometric structures modeled on precisely one of the Thurston geometries. In light of the connection that exists between topology, constant curvature metrics, and the spectrum in dimension two (see p. 876), it becomes tempting to consider the more difficult question of the degree to which the spectrum detects the special relationship that exists between the topology of closed threemanifolds and locally homogeneous metrics.

3.2. Towards hearing the fundamental building blocks of three-dimensional space. The three-dimensional symmetry maximal geometries have been classified by Thurston.

Theorem 14 (Thurston). A three-dimensional symmetry maximal geometry is equivalent to one of the following geometries:

(T1) $\left(\mathbb{R}^{3}\right.$, Isom $\left.\left(\mathbb{E}^{3}\right)^{o}\right)$,

(T2) $\left(S^{3}, \operatorname{Isom}\left(\mathbb{S}^{3}\right)^{o}\right)$,

(T3) $\left(\mathbb{M}^{3}, \operatorname{Isom}\left(\mathbb{M}^{3}\right)^{o}\right)$,

(T4) $\left(S^{2} \times \mathbb{R}, \operatorname{Isom}\left(\mathbb{S}^{2} \times \mathbb{E}\right)^{o}\right)$,

(T5) $\left(\mathbb{\boxplus}^{2} \times \mathbb{E}, \operatorname{Isom}\left(\mathbb{R}^{2} \times \mathbb{E}\right)^{o}\right)$,

(T6) (Nil, Isom(Nil, $\left.\left.g_{\max }\right)^{o}\right)$,

(T7) $\left(\widetilde{\mathrm{SL}_{2}(\mathbb{R})}, \operatorname{Isom}\left(\widetilde{\mathrm{SL}_{2}(\mathbb{R})}, g_{\max }\right)^{o}\right)$,

(T8) $\left(\operatorname{Sol}, \operatorname{Isom}\left(\operatorname{Sol}, g_{\max }\right)^{o}\right)$,

where $\mathrm{Nil}$ (respectively, $\widetilde{\mathrm{SL}_{2}(\mathbb{R})}$ or $\left.\mathrm{Sol}\right)$ is a particular threedimensional unimodular Lie group and $g_{\max }$ is a left-invariant metric with the property that $\operatorname{Isom}\left(g_{\max }\right)^{\circ}$ is maximal among all left-invariant metrics on $\mathrm{Nil}$ (respectively, $\widetilde{\mathrm{SL}_{2}(\mathbb{R})}$ or $\mathrm{Sol}$ ).

The preceding geometries are known collectively as the Thurston geometries and, as we discussed at the end of the

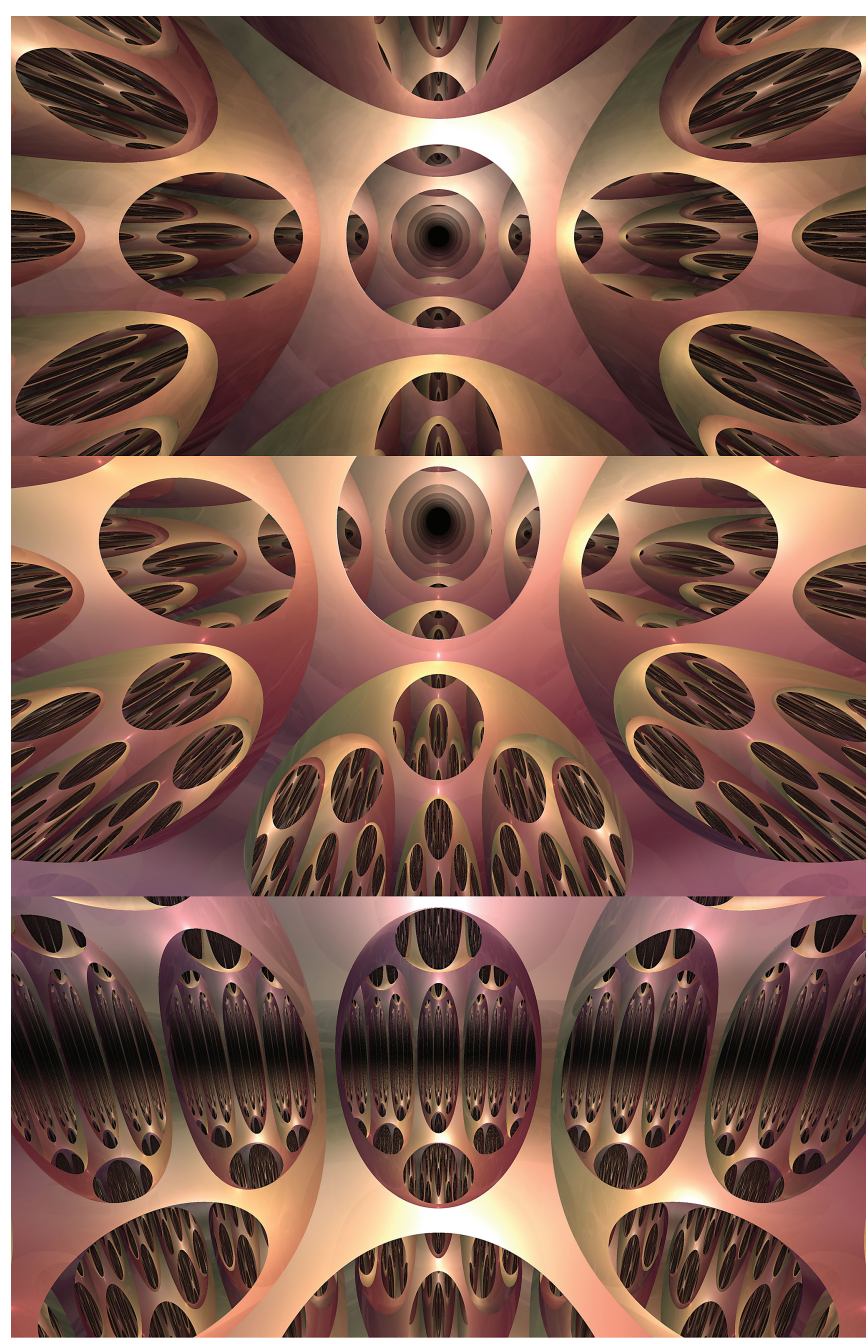

Figure 6. $\mathbb{U}^{2} \times \mathbb{E}^{1}$ is one of the eight Thurston geometries (see Theorem 14). There are numerous examples of isospectral manifolds modeled on this geometry and, among locally homogeneous spaces, Riemannian manifolds modeled on $\mathbb{Q}^{2} \times \mathbb{E}^{1}$ are spectrally determined up to Riemannian cover (see Theorem 16). Here we see images of a cubical tiling of $\mathbb{M}^{2} \times \mathbb{S}^{1}$. The first view is along the $\mathbb{S}^{1}$ factor, the second is half way between the two factors, and the final image is parallel to the $\mathbb{T}^{2}$ factor.

previous section, they can be thought of as the fundamental building blocks of three-dimensional space. Additionally, Sekigawa has classified the simply-connected homogeneous spaces that admit compact quotients [Sek] and by combining this with the work of Raymond and Vasquez (1981, MR0612013), we obtain the following classification of three-dimensional metrically maximal geometries.

Theorem 15 (See [LSS1]). A compact locally homogeneous three-manifold is modeled on one of the following metrically maximal geometries:

(MM1) the $\widetilde{\mathrm{E}(2)}$-geometry $(\widetilde{\mathrm{E}(2)}, \widetilde{\mathrm{E}(2)})$,

(MM2) the $S^{3}$-geometry $\left(S^{3}, S^{3}\right)$, 


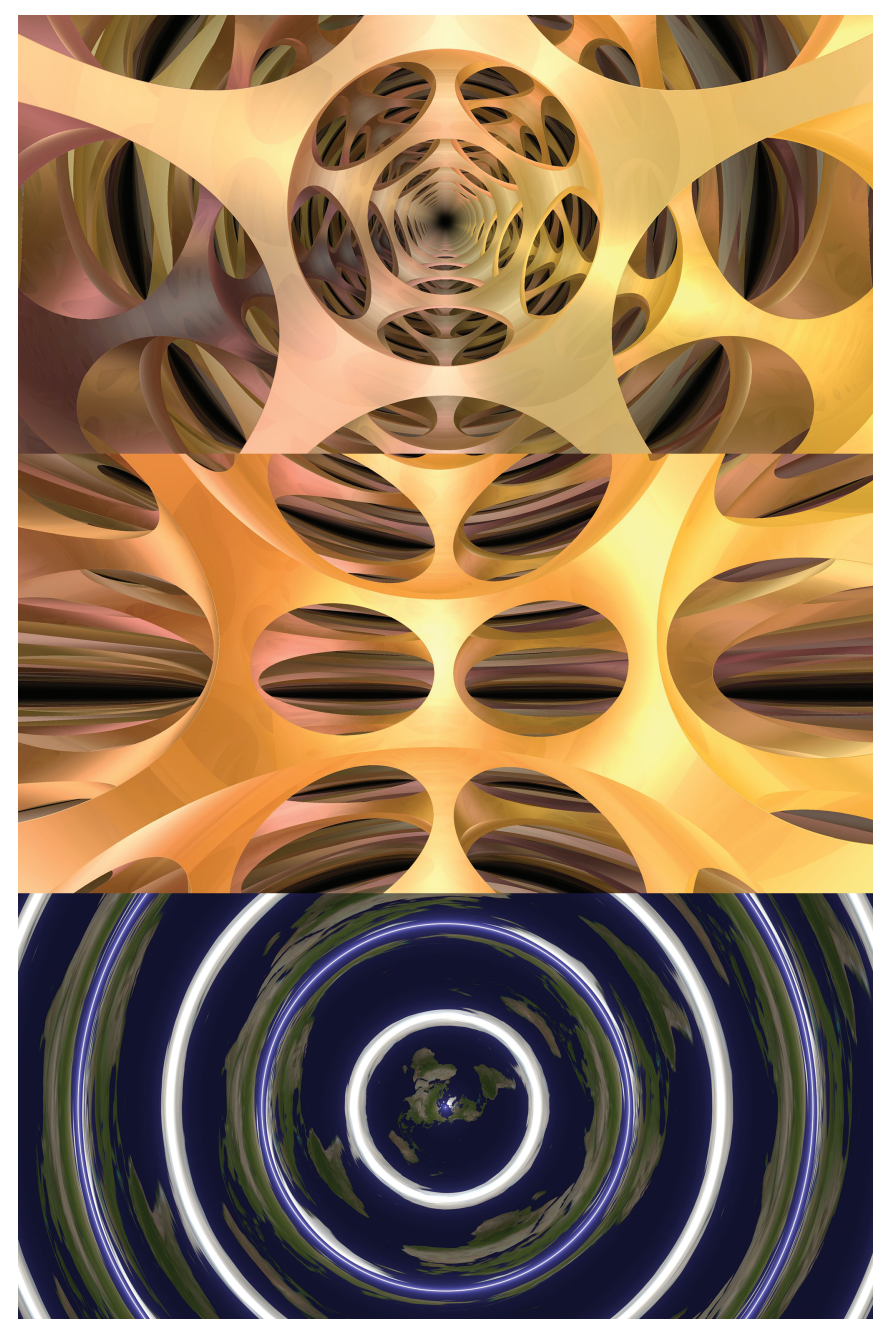

Figure 7. Spaces modeled on the geometry $\mathbb{S}^{2} \times \mathbb{E}(\mathrm{MM} 4)$ are uniquely characterized by their spectra among all locally homogeneous spaces (see Theorem 18), which suggests they are spectrally determined. The first two images depict a tiling of $\mathbb{S}^{2} \times \mathbb{S}^{1}$ formed by drawing a dodecahedron on the $S^{2}$ factor and placing a sphere on each face. The first view is along the $\mathbb{S}^{1}$ factor, while the second is parallel to the $\mathbb{S}^{2}$ factor. The third image shows the surface $\mathbb{S}^{2} \times\{0\}$ in $\mathbb{S}^{2} \times \mathbb{E}$ as viewed along the Euclidean factor. The 2-sphere $\mathbb{S}^{2}$ has been textured as the earth to enable the viewer to follow where the geodesics from their eye land on the surface $\mathbb{S}^{2} \times\{0\}$.

(MM3) the $\mathbb{M}^{3}$-geometry $\left(\mathbb{M}^{3}, \operatorname{Isom}\left(\mathbb{M}^{3}\right)^{o}\right)$,

$(\mathrm{MM} 4)$ the $\mathbb{S}^{2} \times \mathbb{E}$-geometry $\left(S^{2} \times \mathbb{R}, \operatorname{Isom}\left(\mathbb{S}^{2} \times \mathbb{E}\right)^{o}\right)$,

(MM5) the $\mathbb{\boxplus}^{2} \times \mathbb{R}$-geometry $\left(\mathbb{M}^{2} \times \mathbb{E}\right.$, Isom $\left.\left(\mathbb{M}^{2} \times \mathbb{E}\right)^{o}\right)$,

(MM6) the Nil-geometry (Nil, Nil),

(MM7) the $\widetilde{\mathrm{SL}_{2}(\mathbb{R})}$-geometry $\left(\widetilde{\mathrm{SL}_{2}(\mathbb{R})}, \widetilde{\mathrm{SL}_{2}(\mathbb{R})}\right)$, or

(MM8) the Sol-geometry (Sol, Sol),

where $\widetilde{\mathrm{E}(2)}$ is the universal cover of $\operatorname{Isom}\left(\mathbb{E}^{2}\right)^{o}$ and for any unimodular Lie group $G$, the geometry $(G, G)$ is given by $G$ acting on itself by left multiplication.

Each of the metrically maximal geometries above is contained in a distinct and unique symmetry maximal geometry: for $j=1, \ldots, 8,(\mathrm{MM} j)$ is contained in $(\mathrm{T} j)$ with equality if and only if $j=3,4$, or 5 .

The existence of isospectral, yet non-isometric, hyperbolic three-manifolds shows the most general result possible in dimension three is an affirmative answer to Problem 3(c) for any three-dimensional model geometry $(X, G)$. In joint work with $S$. Lin and B. Schmidt that utilizes the first four heat invariants (i.e., $a_{0}, a_{1}, a_{2}$, and $a_{3}$ ), we have recently provided evidence that this is the case.

Theorem 16 (Lin, Schmidt, and Sutton [LSS1]). Let $(M, g)$ and $(N, h)$ be two isospectral compact locally homogeneous three-manifolds.

1. Suppose $(M, g)$ is modeled on one of the following metrically maximal geometries (MM1) or (MM3)-(MM6). Then, $(M, g)$ and $(N, h)$ have isometric universal Riemannian coverings.

2. Suppose $(M, g)$ is modeled on $(\mathrm{MM} 2)$. Then $(N, h)$ is also modeled on (MM2). Furthermore, if $g$ is sufficiently close to a metric of constant sectional curvature, then $(M, g)$ and $(N, h)$ have isometric universal Riemannian coverings.

3. Suppose $(M, g)$ and $(N, h)$ are both modeled on (MM8). Then they have isometric universal Riemannian coverings.

Momentarily restricting our attention to symmetry maximal geometries, we have the following corollary suggesting the fundamental three-dimensional "building blocks" (i.e., Thurston geometries) are audible.

Corollary 17 (Lin, Schmidt, and Sutton [LSS1]). Let (M, g) be a compact locally homogeneous three-manifold modeled on one of the Thurston geometries (T1)-(T6). Then, among locally homogeneous three-manifolds, $(M, g)$ is spectrally determined up to universal Riemannian cover. In particular, among locally homogeneous spaces, the local geometry of a locally symmetric three-manifold is audible.

As one expects the most likely candidate to share the same spectrum as a locally homogeneous three-manifold is another locally homogeneous three-manifold; our work indicates certain compact locally homogeneous threemanifolds are likely spectrally determined.

Theorem 18 (Lin, Schmidt, and Sutton [LSS1]). Among compact locally homogeneous three-manifolds, a Riemannian manifold modeled on the $\mathbb{S}^{2} \times \mathbb{E}$-geometry (MM4) or the Nilgeometry (MM6) is determined up to isometry by its spectrum.

We note that while there are just four compact manifolds that admit geometric structures modeled on the $\mathbb{S}^{2} \times$ E-geometry (MM4) (see Figure 7), namely, $S^{2} \times S^{1}, \mathbb{R} P^{2} \times$ $S^{1}, \mathbb{R} P^{3} \# \mathbb{R} P^{3}$, and the non-trivial $S^{1}$-bundle over $\mathbb{R} P^{2}$, there are infinitely many compact three-manifolds admitting geometric structures modeled on the Nil-geometry (MM6). 


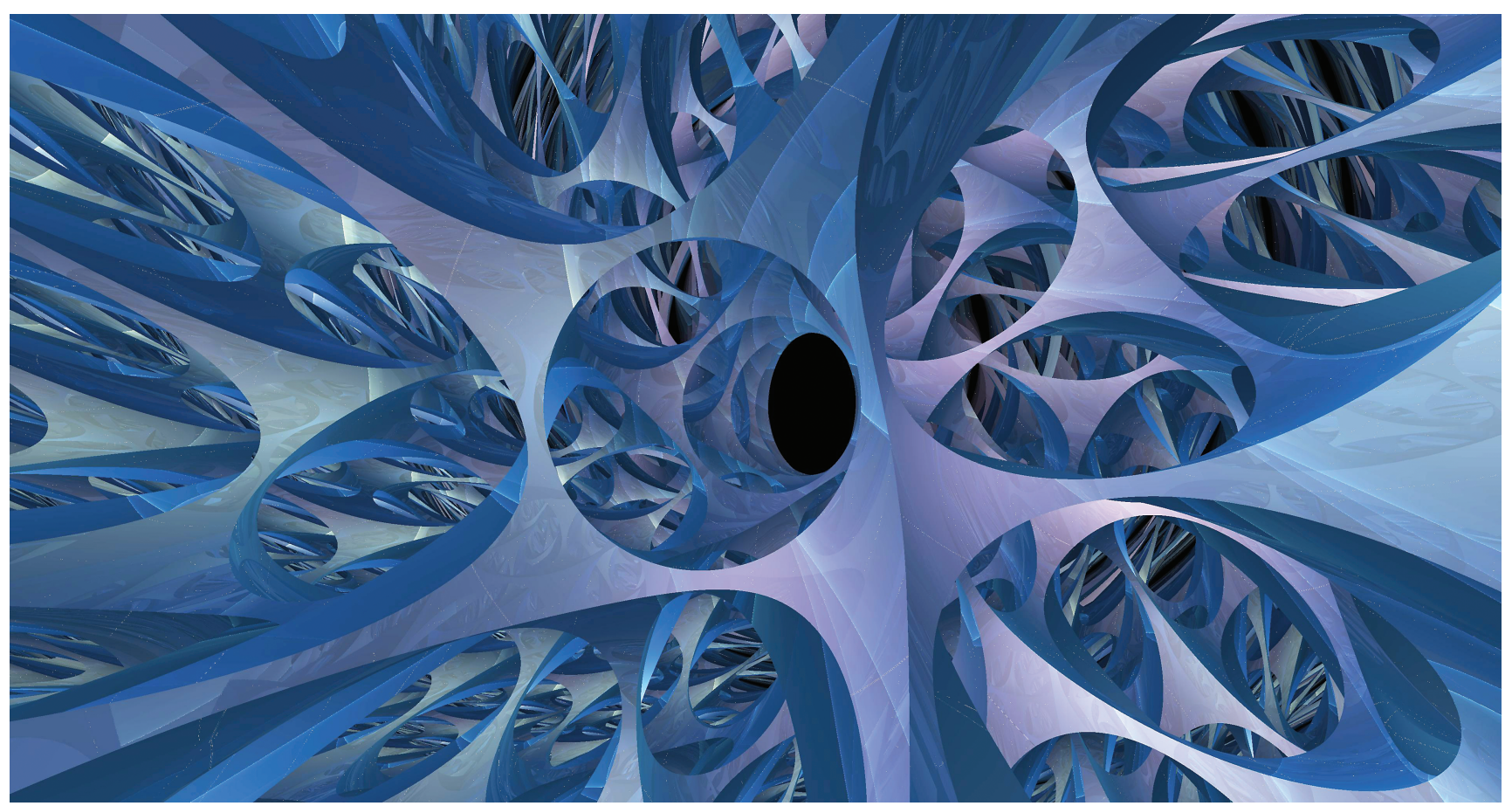

Figure 8. Dehn-twisted torus bundles admit geometric structures modeled on the Nil-geometry (MM6) and, by Theorem 18, such geometric structures are spectrally determined among locally homogeneous spaces, suggesting they are spectrally determined among all Riemannian manifolds. Here we see a cubical tiling of a Dehn-twisted torus bundle.

Example 19 (Dehn-twisted torus bundles and the Nil-geometry). Let $T^{2}=\mathbb{R}^{2} / \mathbb{Z}^{2}$ be the two-torus and $A \in$ $\mathrm{SL}(2, \mathbb{Z})$. Then the closed three-manifold

$$
M_{A} \equiv T^{2} \times[0,1] /\{(\overline{\mathbf{x}}, 0) \sim(\overline{A \mathbf{x}}, 1)\}
$$

is a torus bundle over $S^{1}$ and all torus bundles over $S^{1}$ arise in this fashion. $M_{A}$ is an example of what is commonly known as a mapping torus. Any three-manifold finitely covered by some $M_{A}$, where $A \in \mathrm{SL}(2, \mathbb{Z})$ has $|\operatorname{Tr}(A)|=2$, admits a geometric structure modeled on the Nil-geometry (MM6). Such spaces are precisely the Dehn-twisted torus bundles over $S^{1}$ (see Figure 8 ).

3.3. Hearing the round sphere. To place Problem 3 and the preceding results in context, it is worth keeping in mind that it is still unknown whether the round $n$-sphere, $\left(S^{n}, g_{\text {round }}\right)$, is uniquely determined by its spectrum in every dimension. Of all compact Riemannian manifolds, the round $n$-sphere is arguably the most symmetric. Indeed, it is a globally symmetric space and isotropic, ${ }^{13}$ modeled on a symmetry maximal geometry and the dimension of its isometry group is the largest possible among all $n$ manifolds. Closely related to this fact, the eigenvalues of the associated Laplace operator occur with extremely large

\footnotetext{
${ }^{13} A$ Riemannian manifold $(M, g)$ is said to be isotropic if for any $p \in M$ and $v, w \in T_{p} M$ there is an isometry $f \in \operatorname{Isom}(M, g)$ such that $f_{*}(v)=w$. All isotropic manifolds are homogeneous; therefore, the geometry looks the same at all points and in all directions. It is known that a connected isotropic Riemannian manifold is either flat or a symmetric space of rank one.
}

multiplicities. As a consequence, it is widely expected that the round $n$-sphere is determined up to isometry by its spectrum. In spite of the plausibility of this claim, it is only known to be true in dimension six or less, as was demonstrated by Tanno through the use of the heat invariants $a_{0}$, $a_{1}$, and $a_{2}$ [T1]. Subsequently, Tanno used the heat invariants to demonstrate that the round metric is spectrally isolated within $\mathscr{R}\left(S^{n}\right)$ for all $n$ [T2]. As we noted earlier, Gordon's examples of isospectral deformations on spheres of dimension eight or higher (Theorem 9) come arbitrarily close to the round metric, so Tanno's spectral isolation result for the round sphere is unstable. Analogous spectral isolation results for flat metrics and metrics of constant negative sectional curvature can be found in the work of R. Kuwabara (1980, MR0593057) and V. Sharafutdinov (2009, MR2603860), respectively.

We conclude by remarking that Bettiol, Lauret, and Piccione (2020, arXiv:2001.08471) have recently provided additional evidence that the round sphere is uniquely determined by its spectrum. Through the explicit computation of the fundamental tone $\lambda_{1}$, they have demonstrated that two homogeneous spheres are isospectral if and only if they are isometric, thus eliminating the most likely source of counterexamples. Since an evendimensional sphere has a unique (up to scaling) homogeneous metric (i.e., the metric of constant sectional curvature +1), this result provides no new insight in even dimensions. However, odd-dimensional spheres admit 


\begin{tabular}{|c|c|c|c|}
\hline$(X, G)$ & Audible up to Model Geometry & Audible up to Universal Riemannian Cover & Audible \\
\hline$(\widetilde{E(2)}, \widetilde{E(2)})$ & Yes & Yes & $\begin{array}{l}\text { Yes, if }(M, g) \text { is flat } \\
\text { and not "Tetra" or "Didi"; } \\
\text { otherwise, it is unknown }\end{array}$ \\
\hline$\left(S^{3}, S^{3}\right)$ & Yes & $\begin{array}{l}\text { Yes, if }(M, g) \text { is close to constant sectional } \\
\text { curvature; otherwise, it is unknown }\end{array}$ & $\begin{array}{l}\text { Yes, if }(M, g) \text { has constant } \\
\text { sectional curvature; } \\
\text { otherwise, it is unknown }\end{array}$ \\
\hline$\left(\mathbb{M}^{3}, \operatorname{Isom}\left(\mathbb{M}^{3}\right)^{o}\right)$ & Yes & Yes & $\begin{array}{l}\text { No, many non-trivial } \\
\text { isospectral pairs }\end{array}$ \\
\hline$\left(\mathbb{Q}^{2} \times \mathbb{R}, \operatorname{Isom}\left(\mathbb{M}^{2} \times \mathbb{E}\right)^{o}\right)$ & Yes & Yes & $\begin{array}{l}\text { No, many non-trivial } \\
\text { isospectral pairs }\end{array}$ \\
\hline$\left(\mathbb{S}^{2} \times \mathbb{R}, \operatorname{Isom}\left(\mathbb{S}^{2} \times \mathbb{E}\right)^{o}\right)$ & Yes & Yes & Yes \\
\hline (Nil, Nil) & Yes & Yes & Yes \\
\hline$\left(\widetilde{\mathrm{SL}_{2}(\mathbb{R})}, \widetilde{\mathrm{SL}_{2}(\mathbb{R})}\right)$ & $\begin{array}{l}\text { Yes, if }(M, g) \text { is symmetry maximal } \\
\text { or under certain conditions } \\
\text { on } \operatorname{Ric}(g) \text {; otherwise, it is unknown }\end{array}$ & $\begin{array}{l}\text { Yes, under further conditions on } \operatorname{Ric}(g) \text {; } \\
\text { otherwise, it is unknown }\end{array}$ & $? ?$ \\
\hline (Sol, Sol) & ?? & Yes, among spaces modeled on (Sol, Sol) & $? ?$ \\
\hline
\end{tabular}

Figure 9. The degree to which a three-dimensional compact geometric structure $(M, g)$ modeled on $(X, G)$ can be distinguished from other compact locally homogeneous three-manifolds via its spectrum (see [LSS1]). We note that "Tetra and Didi" form the unique (up to scaling) flat isospectral pair in dimension three, an example constructed by Doyle and Rossetti (2004, MR2087082).

homogeneous metrics beside the round metrics. Indeed, the left-invariant metrics on $S^{3} \simeq \mathrm{SU}(2)$ and the Hopf fibrations $S^{1} \hookrightarrow S^{2 n+1} \rightarrow \mathbb{C} P^{n}, S^{3} \hookrightarrow S^{4 n+3} \rightarrow \mathbb{H} P^{n}$, and $S^{7} \hookrightarrow S^{15} \rightarrow S^{8}$ each gives rise to three-parameter, twoparameter, four-parameter, and two-parameter families of homogeneous metrics on $S^{3}, S^{2 n+1}, S^{4 n+3}$, and $S^{15}$, respectively. These families of metrics correspond to the metrically maximal geometries $\left(S^{3}, S^{3}\right),\left(S^{2 n+1}, \mathrm{SU}(n+1)\right)$ for $n \geq 2,\left(S^{4 n+1}, \operatorname{Sp}(n+1)\right)$ for $n \geq 1$, and $\left(S^{15}, \operatorname{Spin}(9)\right)$, respectively. And, it was shown by W. Ziller (1982, MR0661203) that, up to isometry, the preceding discussion accounts for all the homogeneous metrics on standard spheres.

\section{References}

[AYY] J. An, J.-K. Yu, and J. Yu, On the dimension datum of a subgroup and its applications to isospectral manifolds, J. Differential Geom. 95 (2013), 59-85, DOI 10.4310/jdg/1361889061. MR3031860

[Bu] P. Buser, Geometry and spectra of compact Riemann surfaces, Progress in Mathematics 106, Birkäuser Boston, Inc., Boston, MA, 1992, xiv+454 pp., DOI 10.1007/978-0-81764992-0. MR1183224.

[DGS] B. De Smit, R. Gornet, and C. Sutton, Sunada's method and the covering spectrum, J. Differential Geom. 86 (2010), 501-537, DOI 10.4310/jdg/1303219428. MR2785841

[Go1] C. S. Gordon, Isospectral closed Riemannian manifolds which are not locally isometric, J. Differential Geom. 37 (1993), 639-649, DOI 10.4310/jdg/1214453902. MR1217163

[Go2] C. S. Gordon, Isospectral deformations of metrics on spheres, Invent. Math. 145 (2001), 317-331, DOI 10.1007/s002220100150. MR1872549
[GWW] C. Gordon, D. Webb, and S. Wolpert, Isospectral plane domains and surfaces via Riemannian orbifolds, Invent. Math. 110 (1992), no. 1, 1-22, DOI 10.1007/BF01231320 MR1181812

[GoWi] C. S. Gordon and E. Wilson, Continuous families of isospectral Riemannian metrics which are not locally isometric, J. Differential Geom. 47 (1997), 504-529, DOI 10.4310/jdg/1214460548. MR1617640

[Ka] M. Kac, Can one hear the shape of a drum?, Amer. Math. Month. 73 (1966), no. 4, part II, 1-23, DOI 10.2307/2313748. MR0201237

[LP] M. Larsen and R. Pink, Determining representations from invariant dimensions, Invent. Math. 102 (1990), 377-398, DOI 10.1007/BF01233432. MR1074479

[LSS1] S. Lin, B. Schmidt, and C. Sutton, Geometric structures and the Laplace spectrum, arXiv: 1905 .11454[math.DG], preprint.

[LSS2] S. Lin, B. Schmidt, and C. Sutton, Geometric structures and the Laplace spectrum, part II, arXiv: 1910 .14118[math.DG], preprint.

[Sc] D. Schueth, Continuous families of isospectral metrics on simply-connected manifolds, Ann. Math. 149 (1999), 287308, DOI 10.2307/121026. MR1680563

[Sek] Kouei Sekigawa, On some 3-dimensional curvature homogeneous spaces, Tensor (N.S.) 31 (1977), no. 1, 87-97. MR464115

[Su] T. Sunada, Riemannian coverings and isospectral manifolds, Ann. Math. 121 (1985), 169-186, DOI 10.2307/1971195. MR0782558

[Sut] C. J. Sutton, Isospectral simply-connected homogeneous spaces and the spectral rigidity of group actions, Comment. Math. Helv. 77 (2002), 701-717, DOI 10.1007/PL00012438. MR1949110 
[Sz] Z. Szabó, Locally non-isometric yet super isospectral spaces, Geom. Funct. Anal. 9 (1999), 185-214, DOI 10.1007/s000390050084, MR1675894

[T1] S. Tanno, Eigenvalues of the Laplacian of Riemannian manifolds, Tohoku Math. J. (2) 25 (1973), 391-403, DOI 10.2748/tmj/1178241341. MR0334086

[T2] S. Tanno, A characterization of the canonical spheres by the spectrum, Math. Z. 175 (1980), 267-274, DOI 10.1007/BF01163028. MR0602639

[Yu] J. Yu, On dimension datum of a subgroup, Duke Math. J. 165 (2016), 2683-2736, DOI 10.1215/001270943619898, MR3551771

[Ze] Steve Zelditch, Survey on the inverse spectral problem, ICCM Not. 2 (2014), no. 2, 1-20, DOI 10.4310/ICCM.2014.v2.n2.a1. MR3314780

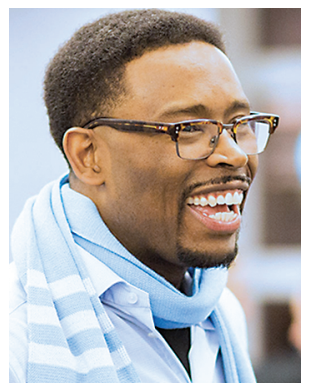

\section{Craig Sutton}

\section{Credits}

Opening image and Figures 5, 6, 7, and 8 are courtesy of Steve Trettel.

Photo of the author is courtesy of Dartmouth College/Joshua Renaud.

\section{New from the European Mathematical Society}

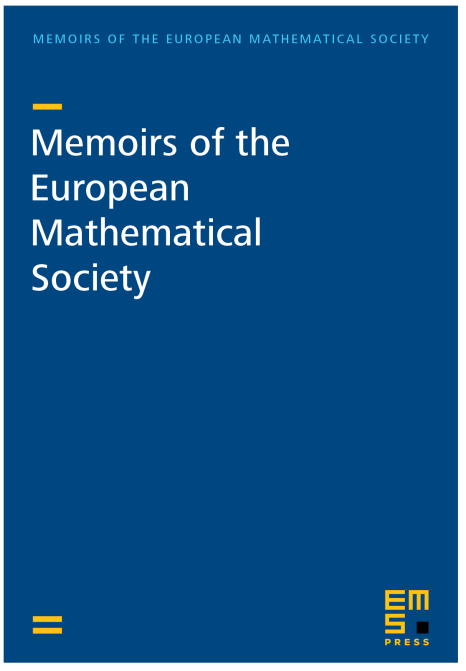

ISSN print 2747-9080 ISSN online 2747-9099

ems.press/mems

The Memoirs of the European Mathematical Society publish outstanding research contributions in individual volumes, in all areas of mathematics and with a particular focus on works that are longer and more comprehensive than usual research articles.

The Editorial Board consists of the Editors-in-Chief of the Journal of the European Mathematical Society and the EMS Surveys in Mathematical Sciences, along with editors of book series published by EMS Press.

Publication begins in January 2022. Each volume will be available individually or as part of an annual subscription.

Submissions are now open. All submitted works will go through a highly selective peer-review process.

For more details visit ems.press/mems

\section{Editorial Board}

Gerard van der Geer | Universiteit van Amsterdam Barbara Kaltenbacher | Universität Klagenfurt

Thomas Kappeler | Universität Zürich

Ari Laptev | Imperial College, London

François Loeser | Sorbonne Université, Paris

Laure Saint-Raymond | École Normale Supérieure de Lyon

EMS Press is an imprint of the European Mathematical Society - EMS - Publishing House GmbH | Straße des 17. Juni 136| 10623 Berlin, Germany | https://ems. press
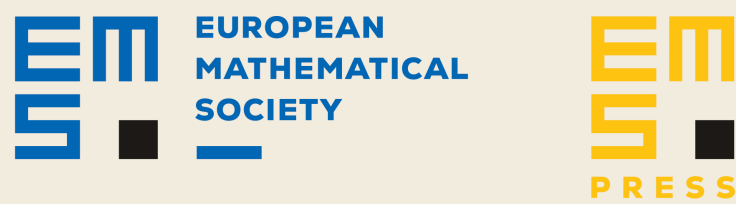